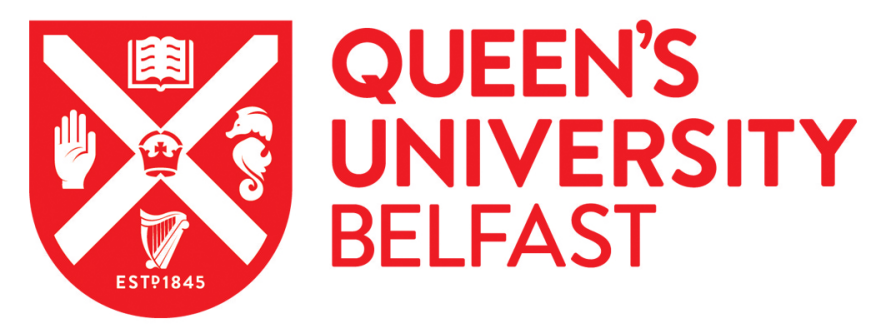

\title{
Orthometallation in iridium $\mathrm{N}$-heterocycle stabilized carbene complexes via carbonhalogen and carbonhydrogen bond activation induced by silver nanoparticles
}

Marr, A. C., Saunders, G. C., Thomas, H. P., \& Wang, Y. (2019). Orthometallation in iridium N-heterocycle stabilized carbene complexes via carbonhalogen and carbonhydrogen bond activation induced by silver nanoparticles. Inorganica Chimica Acta, 486, 1-7. https://doi.org/10.1016/j.ica.2018.10.016

Published in:

Inorganica Chimica Acta

Document Version:

Peer reviewed version

Queen's University Belfast - Research Portal:

Link to publication record in Queen's University Belfast Research Portal

\section{Publisher rights}

Copyright 2018 Elsevier B. V. All rights reserved.

This manuscript is distributed under a Creative Commons Attribution-NonCommercial-NoDerivs License

(https://creativecommons.org/licenses/by-nc-nd/4.0/), which permits distribution and reproduction for non-commercial purposes, provided the author and source are cited.

\section{General rights}

Copyright for the publications made accessible via the Queen's University Belfast Research Portal is retained by the author(s) and / or other copyright owners and it is a condition of accessing these publications that users recognise and abide by the legal requirements associated with these rights.

\section{Take down policy}

The Research Portal is Queen's institutional repository that provides access to Queen's research output. Every effort has been made to ensure that content in the Research Portal does not infringe any person's rights, or applicable UK laws. If you discover content in the

Research Portal that you believe breaches copyright or violates any law, please contact openaccess@qub.ac.uk. 
Orthometallation in Iridium $N$-Heterocycle Stabilized Carbene Complexes via Carbon-Halogen and Carbon-Hydrogen Bond Activation induced by Silver Nanoparticles

Andrew C. Marr ${ }^{a}$, Graham C. Saunders ${ }^{b} *$, Hayden P. Thomas ${ }^{b}$ and Yue-Ming Wang ${ }^{a}$

${ }^{a}$ School of Chemistry and Chemical Engineering, Queen's University Belfast, Belfast BT9 5AG, United Kingdom

${ }^{b}$ School of Science, University of Waikato, Private Bag 3105, Hamilton 3240, New Zealand

Dedicated to Malcolm Green FRS, for his inspiration and guidance. 


\section{Abstract}

On treatment with silver nanoparticles $\left[\mathrm{Cp}^{*} \mathrm{IrCl}_{2}\left(\kappa \mathrm{C}-\mathrm{MeNC}_{3} \mathrm{H}_{2} \mathrm{NCH}_{2} \mathrm{C}_{6} \mathrm{H}_{4} \mathrm{~F}-2\right)\right]$ underwent $\mathrm{C}-\mathrm{H}$ bond activation to afford the orthometallated product $\left[\mathrm{Cp} * \mathrm{IrCl}\left(\kappa \mathrm{C}^{2}-\mathrm{MeNC}_{3} \mathrm{H}_{2} \mathrm{NCH}_{2} \mathrm{C}_{6} \mathrm{H}_{3} \mathrm{~F}-6\right)\right]$ exclusively. The same product was obtained from $\left[\mathrm{Cp}^{*} \mathrm{IrCl}_{2}\left(\kappa \mathrm{C}-\mathrm{MeNC}_{3} \mathrm{H}_{2} \mathrm{NCH}_{2} \mathrm{C}_{6} \mathrm{H}_{4} \mathrm{~F}_{2}-2,6\right)\right]$ by $\mathrm{C}-\mathrm{F}$ bond activation. Treatment of $\left[\mathrm{Cp}^{*} \mathrm{IrCl}_{2}\left(\kappa \mathrm{C}-\mathrm{MeNC}_{3} \mathrm{H}_{2} \mathrm{NCH}_{2} \mathrm{C}_{6} \mathrm{H}_{3} \mathrm{~F}-2-\mathrm{X}-6\right)\right](\mathrm{X}=\mathrm{Cl}, \mathrm{Br}, \mathrm{I})$ with silver nanoparticles induced $\mathrm{C}-\mathrm{F}$ and $\mathrm{C}-\mathrm{X}$ bond activation to yield mixtures of the orthometallated products $\left[\mathrm{Cp} * \operatorname{IrCl}\left(\kappa \mathrm{C}^{2}-\mathrm{MeNC}_{3} \mathrm{H}_{2} \mathrm{NCH}_{2} \mathrm{C}_{6} \mathrm{H}_{3} \mathrm{~F}-6\right)\right]$ and $\left[\mathrm{Cp} * \operatorname{IrCl}\left(\kappa \mathrm{C}^{2}-\mathrm{MeNC}_{3} \mathrm{H}_{2} \mathrm{NCH}_{2} \mathrm{C}_{6} \mathrm{H}_{3} \mathrm{X}-6\right)\right]$. The structures of the products, which are isostructural and co-crystallized, have been determined by single crystal X-ray diffraction. 


\section{Introduction}

There have been a number of reports of $\left[\mathrm{Cp}^{*} \operatorname{IrX}{ }_{2}(\mathrm{NHC})\right]$ complexes undergoing cyclometallation reactions involving aromatic [1-4], aliphatic [2,5,6] and carborane carbonhydrogen bond activation [7]. Although an early example, involving [Cp* $\left.\mathrm{IrCl}_{2}\left(\kappa \mathrm{C}-\mathrm{MeNC}_{3} \mathrm{H}_{2} \mathrm{NCH}_{2} \mathrm{C}_{6} \mathrm{H}_{5}\right)\right]$, was reported to occur spontaneously [1], the reaction is typically induced by base, although acid induced $\mathrm{C}-\mathrm{H}$ bond activation leading to a putative cyclometallated intermediate has also been reported [8].

Recently we reported the orthometallation of $\left[\mathrm{Cp} * \mathrm{IrCl}_{2}\left(\kappa \mathrm{C}-\mathrm{MeNC}_{3} \mathrm{H}_{2} \mathrm{NCH}_{2} \mathrm{C}_{6} \mathrm{~F}_{5}\right)\right]$ via carbon-fluorine bond activation on treatment with silver particles or nanoparticles [9]. In order to determine whether the combination of $\left[\mathrm{Cp}^{*} \operatorname{IrX} \mathrm{X}_{2}(\mathrm{NHC})\right]$ and silver particles could also activate carbon-hydrogen and other carbon-halogen bonds we chose to investigate the 2-fluorophenylmethyl and 2-halo-6-fluorophenylmethyl analogues. Here we report the results of our study.

\section{Experimental}

\subsection{Instrumentation}

The ${ }^{1} \mathrm{H},{ }^{13} \mathrm{C}\left\{{ }^{1} \mathrm{H}\right\}$ and ${ }^{19} \mathrm{~F}$ NMR spectra were recorded in deuterochloroform, except where stated otherwise, using Bruker DRX300 or DPX400 spectrometers. ${ }^{1} \mathrm{H}$ (300.13 or $400.14 \mathrm{MHz}$ ) were referenced internally using the residual proto solvent resonance relative to

$\mathrm{SiMe}_{4}(\delta 0),{ }^{13} \mathrm{C}(75.48$ or $100.61 \mathrm{MHz})$ externally to $\mathrm{SiMe}_{4}(\delta 0)$, and ${ }^{19} \mathrm{~F}(282.40 \mathrm{MHz})$ externally to $\mathrm{CFCl}_{3}(\delta 0)$. All chemical shifts are quoted in $\delta(\mathrm{ppm})$, using the high frequency positive convention, and coupling constants in Hz. Elemental analyses were carried out by the Campbell Microanalytical Laboratory, The University of Otago. Electrospray mass spectra were recorded on a Bruker Daltonics micrOTOF spectrometer. 


\subsection{Materials}

The imidazolium salts $\left[\mathrm{MeNC}_{3} \mathrm{H}_{3} \mathrm{NCH}_{2} \mathrm{C}_{6} \mathrm{H}_{3} \mathrm{X}-2-\mathrm{F}-6\right] \mathrm{Br}, \mathrm{X}=\mathrm{F}, \mathrm{Cl}, \mathrm{Br}$ and I [10], and $\left[\mathrm{Cp}^{*} \operatorname{IrCl}(\mu-\mathrm{Cl})\right]_{2}[11]$ were prepared as previously described. 1-Methylimidazole, silver(I) oxide (Aldrich) and 2-fluorobenzyl bromide (Apollo Scientific) were used as supplied.

\subsection{Preparation of 1-(2-fluorophenylmethyl)-3-methylimidazolium bromide}

1-Methylimidazole (1.55 g, $18.6 \mathrm{mmol}$ ) was added to 2-fluorobenzyl bromide (3.52 g, 18.6 mmol) in dichloromethane $\left(80 \mathrm{~cm}^{3}\right)$. The mixture was left at ambient temperature for $24 \mathrm{~h}$. The solvent was removed by rotary evaporation yielding the product as a pale yellow oil. Yield ca. 5 g $100 \%$. $[\mathrm{M}-\mathrm{Br}]^{+}:\left[\mathrm{C}_{11} \mathrm{H}_{12} \mathrm{FN}_{2}\right]^{+}$requires 191.0985; found 191.1032. $\delta_{\mathrm{H}}$ $\left(\mathrm{CDCl}_{3} /\left(\mathrm{CD}_{3}\right)_{2} \mathrm{SO}\right): 9.89\left(1 \mathrm{H}, \mathrm{s}, \mathrm{N}_{2} \mathrm{CH}\right), 7.63\left(1 \mathrm{H}, \operatorname{tm} J=7.6 \mathrm{~Hz}, \mathrm{C}_{6} \mathrm{H}\right), 7.59(1 \mathrm{H}, \mathrm{dd}, J=4.7$, $\left.1.7 \mathrm{~Hz}, \mathrm{C}_{6} \mathrm{H}\right), 7.49\left(1 \mathrm{H}, \mathrm{dd}, J=4.2,1.4 \mathrm{~Hz}, \mathrm{C}_{6} \mathrm{H}\right), 7.34(1 \mathrm{H}, \mathrm{m}, H \mathrm{CCH}), 7.13(1 \mathrm{H}, \mathrm{m}$, $\mathrm{HCCH}), 7.05\left(1 \mathrm{H}, \mathrm{tm}, J=9.2 \mathrm{~Hz}, \mathrm{C}_{6} \mathrm{H}\right), 5.55\left(2 \mathrm{H}, \mathrm{s}, \mathrm{CH}_{2}\right), 3.96\left(3 \mathrm{H}, \mathrm{d}, J=1.2 \mathrm{~Hz}, \mathrm{CH}_{3}\right) . \delta_{\mathrm{F}}$ $\left(\mathrm{CDCl}_{3} /\left(\mathrm{CD}_{3}\right)_{2} \mathrm{SO}\right):-117.34\left(1 \mathrm{~F}, \mathrm{dd}, J_{\mathrm{HF}}=8.3,6.9 \mathrm{~Hz}\right)$.

\subsection{Preparation of ( $\kappa C-1-(2-f l u o r o p h e n y l m e t h y l)-3-m e t h y l i m i d a z o l-2-y l i d e n e)$ silver bromide} (1a)

Silver(I) oxide $(0.121 \mathrm{~g}, 0.5210 \mathrm{mmol})$ was added to a solution of 1-(2-fluorophenylmethyl)-3-methylimidazolium bromide $(0.215 \mathrm{~g}, 0.794 \mathrm{mmol})$ in dichloromethane $\left(30 \mathrm{~cm}^{3}\right)$. The mixture was stirred in the absence of light at room temperature for $2 \mathrm{~h}$. The reaction mixture was filtered through celite $(2.5 \mathrm{~cm})$, which was subsequently washed with dichloromethane $\left(10 \mathrm{~cm}^{3}\right)$. The solvent was removed from the combined filtrate and washings by rotary evaporation to give yield a yellow-brown oil. The oil was dissolved in chloroform $\left(5 \mathrm{~cm}^{3}\right)$ and filtered through celite $(5 \mathrm{~cm})$, which was 
subsequently washed with chloroform $\left(5 \mathrm{~cm}^{3}\right)$. The product was obtained as an off-white solid by addition of petroleum ether (b.p. $60-80^{\circ} \mathrm{C}, 30 \mathrm{~cm}^{3}$ ) to the combined filtrate and washing, and was washed with petroleum ether (b.p. $60-80^{\circ} \mathrm{C}, 30 \mathrm{~cm}^{3}$ ) and dried in air. Yield: $0.211 \mathrm{~g}(70 \%) .\left[2 \mathrm{M}-\mathrm{AgBr}_{2}\right]^{+}:\left[\mathrm{C}_{22} \mathrm{H}_{22}{ }^{107} \mathrm{AgF}_{2} \mathrm{~N}_{4}\right]^{+}$requires 487.0863; found: 487.1346. $\delta_{\mathrm{H}}$ $7.38\left(2 \mathrm{H}, \mathrm{m}, \mathrm{C}_{6} \mathrm{H}_{4}\right), 7.18\left(1 \mathrm{H}, \mathrm{td}, J=7.5,1.2 \mathrm{~Hz}, \mathrm{C}_{6} \mathrm{H}_{4}\right), 7.11(1 \mathrm{H}, \mathrm{ddd}, J=9.8,8.3,1.1 \mathrm{~Hz}$, $\left.\mathrm{C}_{6} \mathrm{H}_{4}\right), 7.05\left(1 \mathrm{H}, \mathrm{dd},{ }^{3} J_{\mathrm{HH}}=1.8 \mathrm{~Hz}, J=0.9 \mathrm{~Hz}, H C C H\right), 6.98\left(1 \mathrm{H}, \mathrm{t},{ }^{3} J_{\mathrm{HH}}=1.8 \mathrm{~Hz}, \mathrm{HCCH}\right)$, $5.35\left(2 \mathrm{H}, \mathrm{s}, \mathrm{CH}_{2}\right), 3.86\left(3 \mathrm{H}, \mathrm{s}, \mathrm{CH}_{3}\right) . \delta_{\mathrm{C}} 182.0(\mathrm{AgC}), 160.7\left(\mathrm{~d},{ }^{1} J_{\mathrm{CF}}=248 \mathrm{~Hz}, \mathrm{CF}\right), 130.9(\mathrm{~d}$, $\left.J_{\mathrm{CF}}=8 \mathrm{~Hz}, \mathrm{C}_{6}\right), 130.7\left(\mathrm{~d}, J_{\mathrm{CF}}=3 \mathrm{~Hz}, \mathrm{C}_{6}\right), 125.0\left(\mathrm{~d}, J_{\mathrm{CF}}=4 \mathrm{~Hz}, \mathrm{C}_{6}\right), 122.8\left(\mathrm{~d}, J_{\mathrm{CF}}=15 \mathrm{~Hz}\right.$, $\left.\mathrm{C}_{6}\right), 122.4(\mathrm{NCH}), 121.4\left(\mathrm{~d}, J_{\mathrm{CF}}=2 \mathrm{~Hz}, \mathrm{NCH}\right), 115.8\left(\mathrm{~d}, J_{\mathrm{CF}}=21 \mathrm{~Hz}, \mathrm{C}_{6}\right), 49.5\left(\mathrm{~s},{ }^{3} J_{\mathrm{CF}}=4\right.$ $\left.\mathrm{Hz}, \mathrm{CH}_{2}\right), 38.9\left(\mathrm{CH}_{3}\right) . \delta_{\mathrm{F}}-117.57(1 \mathrm{~F}, \mathrm{~m})$.

\subsection{Preparation of ( $\kappa C$-1-(2,6-difluorophenylmethyl)-3-methylimidazol-2-ylidene)silver} bromide (1b)

Complex 1b was obtained as a white solid from 1-(2,6-difluorophenylmethyl)-3-methylimidazolium bromide $(0.228 \mathrm{~g}, 0.789 \mathrm{mmol})$ and silver(I) oxide $(0.103 \mathrm{~g}, 0.443 \mathrm{mmol})$ using the procedure in 2.4 . Yield: $0.244 \mathrm{~g}(78 \%)$. [2M$\left.\mathrm{AgBr}_{2}\right]^{+}:\left[\mathrm{C}_{22} \mathrm{H}_{20}{ }^{107} \mathrm{AgF}_{4} \mathrm{~N}_{4}\right]^{+}$requires 523.0675; found: 523.1214. $\delta_{\mathrm{H}}: 7.36\left(1 \mathrm{H}, \mathrm{m}, \mathrm{H}_{\mathrm{para}}\right)$, $7.03\left(1 \mathrm{H}, \mathrm{d},{ }^{3} J_{\mathrm{HH}}=1.8 \mathrm{~Hz}, H \mathrm{CCH}\right), 6.97\left(1 \mathrm{H}, \mathrm{d},{ }^{3} J_{\mathrm{HH}}=1.8 \mathrm{~Hz}, \mathrm{HCCH}\right), 6.96\left(2 \mathrm{H}, \mathrm{m}, \mathrm{H}_{\text {meta }}\right)$, $5.38\left(2 \mathrm{H}, \mathrm{s}, \mathrm{CH}_{2}\right), 3.85\left(3 \mathrm{H}, \mathrm{s}, \mathrm{CH}_{3}\right) . \delta_{\mathrm{C}}: 182.6(\mathrm{AgC}), 161.3\left(\mathrm{dd},{ }^{1} J_{\mathrm{CF}}=251 \mathrm{~Hz},{ }^{3} J_{\mathrm{CF}}=7 \mathrm{~Hz}\right.$, $\mathrm{CF}), 131.4\left(\mathrm{t},{ }^{3} J_{\mathrm{CF}}=11 \mathrm{~Hz}, \mathrm{C}_{\mathrm{para}}\right.$ or $\left.\mathrm{C}_{\mathrm{ipso}}\right), 122.5(\mathrm{CH}), 121.0(\mathrm{CH}), 112.0\left(\mathrm{~m}, \mathrm{C}_{\text {meta }}\right), 111.7(\mathrm{t}$, ${ }^{2} J_{\mathrm{CF}}=19 \mathrm{~Hz}, \mathrm{C}_{\mathrm{para}}$ or $\left.\mathrm{C}_{\mathrm{ipso}}\right), 43.0\left(\mathrm{CH}_{2}\right), 39.0\left(\mathrm{CH}_{3}\right) . \delta_{\mathrm{F}}:-112.87(\mathrm{~m})$. 
Complex 1c was obtained as an off-white solid from

1-(2-chloro-6-fluorophenylmethyl)-3-methylimidazolium bromide (0.275 g, $0.900 \mathrm{mmol})$ and silver(I) oxide $(0.133 \mathrm{~g}, 1.15 \mathrm{mmol})$ using the procedure in 2.4 . Yield: $0.331 \mathrm{~g}(89 \%)$. [2M $\left.\mathrm{AgBr}_{2}\right]^{+}:\left[\mathrm{C}_{22} \mathrm{H}_{20}{ }^{107} \mathrm{Ag}^{35} \mathrm{Cl}^{37} \mathrm{ClF}_{2} \mathrm{~N}_{4}\right]^{+}$requires 557.0180; found: 557.0196. $\delta_{\mathrm{H}}: 7.37(1 \mathrm{H}, \mathrm{td}, J$ $\left.=8.1,5.9 \mathrm{~Hz}, \mathrm{C}_{6} \mathrm{H}_{3}\right), 7.31\left(1 \mathrm{H}, \mathrm{m}, \mathrm{C}_{6} \mathrm{H}_{3}\right), 7.11\left(1 \mathrm{H}, \mathrm{m}, \mathrm{C}_{6} \mathrm{H}_{3}\right), 7.02\left(1 \mathrm{H}, \mathrm{d},{ }^{3} J_{\mathrm{HH}}=1.8 \mathrm{~Hz}\right.$, $H \mathrm{CCH}), 6.97\left(1 \mathrm{H}, \mathrm{d},{ }^{3} J_{\mathrm{HH}}=1.8 \mathrm{~Hz}, \mathrm{HCCH}\right), 5.48\left(2 \mathrm{H}, \mathrm{d},{ }^{4} J_{\mathrm{HF}}=1.8 \mathrm{~Hz}, \mathrm{CH}_{2}\right), 3.86(3 \mathrm{H}, \mathrm{s}$, $\left.\mathrm{CH}_{3}\right) . \delta_{\mathrm{C}}: 182.7(\mathrm{AgC}), 161.8\left(\mathrm{~d},{ }^{1} J_{\mathrm{CF}}=251 \mathrm{~Hz}, \mathrm{CF}\right), 135.9\left(\mathrm{~d}, J_{\mathrm{CF}}=5 \mathrm{~Hz}, \mathrm{C}_{6}\right), 131.4\left(\mathrm{~d}, J_{\mathrm{CF}}\right.$ $\left.=10 \mathrm{~Hz}, \mathrm{C}_{6}\right), 126.1\left(\mathrm{~d}, J_{\mathrm{CF}}=3 \mathrm{~Hz}, \mathrm{C}_{6}\right), 122.3(\mathrm{CH}), 121.2\left(\mathrm{~d} ., J_{\mathrm{CF}}=17 \mathrm{~Hz}, \mathrm{C}_{6}\right), 121.0\left(\mathrm{~d},{ }^{5} J_{\mathrm{CF}}\right.$ $=2 \mathrm{~Hz}, \mathrm{NCH}), 114.8\left(\mathrm{~d}, J_{\mathrm{CF}} 22, \mathrm{NCH}\right), 46.4\left(\mathrm{~d},{ }^{3} J_{\mathrm{CF}} 3, \mathrm{CH}_{2}\right), 39.0\left(\mathrm{CH}_{3}\right) . \delta_{\mathrm{F}}:-110.94(1 \mathrm{~F}, \mathrm{~m})$.

2.7 Preparation of ( $\kappa C-1-(2-b r o m o-6-f l u o r o p h e n y l m e t h y l)-3-m e t h y l i m i d a z o l-2-y l i d e n e)$ silver 1 bromide (1d)

Complex 1d was obtained as a white solid from 1-(2-bromo-6-fluorophenylmethyl)-3-methylimidazolium bromide (0.063 g, $0.273 \mathrm{mmol})$ and silver(I) oxide (0.155 g, $0.444 \mathrm{mmol})$ using the procedure in 2.4. Yield: $0.104 \mathrm{~g}(52 \%)$. [2M $\left.\mathrm{AgBr}_{2}\right]^{+}:\left[\mathrm{C}_{22} \mathrm{H}_{20}{ }^{107} \mathrm{Ag}^{79} \mathrm{Br}^{81} \mathrm{BrF}_{2} \mathrm{~N}_{4}\right]^{+}$requires 644.9170; found: 645.1161. $\delta_{\mathrm{H}}\left(\left(\mathrm{CD}_{3}\right)_{2} \mathrm{SO}\right)$ : $7.55\left(1 \mathrm{H}, \mathrm{dt}, J=7.9,1.0 \mathrm{~Hz}, \mathrm{C}_{6} \mathrm{H}\right), 7.45\left(1 \mathrm{H}, \mathrm{d},{ }^{3} J_{\mathrm{HH}}=1.8 \mathrm{~Hz}, H \mathrm{HCH}\right), 7.40(1 \mathrm{H}, \mathrm{td}, J=8.3$, $\left.6.2 \mathrm{~Hz}, \mathrm{C}_{6} \mathrm{H}\right), 7.39(1 \mathrm{H}, \mathrm{s}$ br, $\mathrm{HCCH}), 7.32\left(1 \mathrm{H}, \mathrm{m}, \mathrm{C}_{6} \mathrm{H}\right), 5.43\left(2 \mathrm{H}, \mathrm{d},{ }^{4} J_{\mathrm{HF}}=1.5 \mathrm{~Hz}, \mathrm{CH}_{2}\right)$, $3.75\left(3 \mathrm{H}, \mathrm{s}, \mathrm{CH}_{3}\right) . \delta_{\mathrm{F}}\left(\left(\mathrm{CD}_{3}\right)_{2} \mathrm{SO}\right):-109.81(1 \mathrm{~F}, \mathrm{~m})$. The ${ }^{13} \mathrm{C}\left\{{ }^{1} \mathrm{H}\right\}$ NMR spectrum could not be obtained because of the low solubility of $\mathbf{1 d}$.

2.8 Preparation of ( $\kappa$ C-1-(2-iodo-6-fluorophenylmethyl)-3-methylimidazol-2-ylidene)silver bromide (1e)

Complex 1e was obtained as a white solid from 1-(2-iodo-6-fluorophenylmethyl)-3-methylimidazolium bromide (0.063 g, $0.273 \mathrm{mmol})$ and 
silver(I) oxide $(0.155 \mathrm{~g}, 0.444 \mathrm{mmol})$ using the procedure in 2.4. Yield: $0.100 \mathrm{~g}(60 \%)$. [2M$\left.\mathrm{AgBr}_{2}\right]^{+}:\left[\mathrm{C}_{22} \mathrm{H}_{20}{ }^{107} \mathrm{AgI}_{2} \mathrm{~F}_{2} \mathrm{~N}_{4}\right]^{+}$requires 738.8796; found: 739.1174. $\delta_{\mathrm{H}}\left(\left(\mathrm{CD}_{3}\right)_{2} \mathrm{SO}\right): 7.78(1 \mathrm{H}$, $\left.\mathrm{dm}, J=7.8 \mathrm{~Hz}, \mathrm{C}_{6} \mathrm{H}\right), 7.45\left(1 \mathrm{H}, \mathrm{d}, J_{\mathrm{HH}}=1.8 \mathrm{~Hz}, H C C H\right), 7.33\left(2 \mathrm{H}, \mathrm{m}, \mathrm{C}_{6} \mathrm{H}\right.$ and $\left.\mathrm{HCCH}\right)$, $7.22\left(1 \mathrm{H}, \mathrm{td}, J=7.9,6.0 \mathrm{~Hz}, \mathrm{C}_{6} \mathrm{H}\right), 5.41\left(2 \mathrm{H}, \mathrm{d},{ }^{4} J_{\mathrm{HF}}=1.8 \mathrm{~Hz}, \mathrm{CH}_{2}\right), 3.76\left(3 \mathrm{H}, \mathrm{s}, \mathrm{CH}_{3}\right) . \delta_{\mathrm{F}}$ $\left(\left(\mathrm{CD}_{3}\right)_{2} \mathrm{SO}\right):-108.27(1 \mathrm{~F}, \mathrm{~m})$. The ${ }^{13} \mathrm{C}\left\{{ }^{1} \mathrm{H}\right\}$ NMR spectrum could not be obtained because of the low solubility of $\mathbf{1 e}$.

\subsection{Preparation of}

( $\eta^{5}$-pentamethylcyclopentadienyl)iridium( $\kappa C-1-(2-f l u o r o p h e n y l m e t h y l)-3-m e t h y l i m i d a z o l-2-y$ lidene) dichloride (2a)

Complex 1a $(0.131 \mathrm{~g}, 0.346 \mathrm{mmol})$ was dissolved in dichloromethane $\left(5 \mathrm{~cm}^{3}\right)$ and filtered through celite into a solution of $\left[\mathrm{Cp}^{*} \operatorname{IrCl}(\mu-\mathrm{Cl})\right]_{2}(0.138 \mathrm{~g}, 0.174 \mathrm{mmol})$ in dichloromethane $\left(30 \mathrm{~cm}^{3}\right)$, giving a yellow solution and white precipitate. The reaction mixture was left for 3 hours in the absence of light. The solution was filtered through celite, which was subsequently washed with dichloromethane $\left(2 \times 10 \mathrm{~cm}^{3}\right)$. The solvent was removed from the combined filtrate and washings by rotary evaporation giving an orange oil, which was dissolved in dichloromethane $\left(2 \mathrm{~cm}^{3}\right)$ and filtered through silica. Removal of the solvent by evaporation gave an orange solid which was recrystallized from chloroform and petroleum ether (b.p. $\left.60-80^{\circ} \mathrm{C}\right)$. Yield: $0.174 \mathrm{~g}(85 \%)$. Anal. Calc. for $\mathrm{C}_{21} \mathrm{H}_{26} \mathrm{Cl}_{2} \mathrm{FIrN}_{2} \cdot{ }_{1}^{1} / 8\left(\mathrm{CHCl}_{3}\right)$ : C,

42.0; H, 4.4; N, 4.6. Found: C, 41.8; H, 4.6; N, 5.2\%. [M - Cl $]^{+}:\left[\mathrm{C}_{21} \mathrm{H}_{26}{ }^{35} \mathrm{ClF}^{193} \mathrm{IrN}_{2}\right]^{+}$ requires 553.1471; found: 553.1342. $\delta_{\mathrm{H}} 7.64\left(1 \mathrm{H}, \mathrm{td} ., J=7.7,1.7 \mathrm{~Hz}, \mathrm{C}_{6} \mathrm{H}_{4}\right), 7.33(1 \mathrm{H}, \mathrm{m}$, $\left.\mathrm{C}_{6} \mathrm{H}_{4}\right), 7.14\left(1 \mathrm{H}, \mathrm{td}, J=7.4,1.2, \mathrm{~Hz}, \mathrm{C}_{6} \mathrm{H}_{4}\right), 7.09\left(1 \mathrm{H}, \mathrm{ddd} ., J=9.5,8.2,1.2 \mathrm{~Hz}, \mathrm{C}_{6} \mathrm{H}_{4}\right), 6.90$ $\left(1 \mathrm{H}, \mathrm{d},{ }^{3} J_{\mathrm{HH}}=2.0 \mathrm{~Hz}, \mathrm{HCCH}\right), 6.69\left(1 \mathrm{H}, \mathrm{t},{ }^{3} J_{\mathrm{HH}}=2.0 \mathrm{~Hz}, \mathrm{HCCH}\right), 6.08\left(1 \mathrm{H}, \mathrm{d},{ }^{2} J_{\mathrm{HH}}=14.5\right.$ $\mathrm{Hz}, \mathrm{NCHH}), 5.30\left(1 \mathrm{H}, \mathrm{d},{ }^{2} J_{\mathrm{HH}}=14.5 \mathrm{~Hz}, \mathrm{NCH} H\right), 4.02\left(3 \mathrm{H}, \mathrm{s}, \mathrm{NCH}_{3}\right), 1.67\left(15 \mathrm{H}, \mathrm{s}, \mathrm{CH}_{3}\right) . \delta_{\mathrm{C}}$ : $160.8\left(\mathrm{~d},{ }^{1} J_{\mathrm{CF}}=246 \mathrm{~Hz}, \mathrm{CF}\right), 156.6\left(\mathrm{IrCN}_{2}\right), 132.0\left(\mathrm{~d}, J_{\mathrm{CF}}=3 \mathrm{~Hz}, \mathrm{C}_{6}\right), 130.1\left(\mathrm{~d}, J_{\mathrm{CF}}=8 \mathrm{~Hz}\right.$, 
$\left.\mathrm{C}_{6}\right), 124.8\left(\mathrm{~d}, J_{\mathrm{CF}}=3 \mathrm{~Hz}, \mathrm{C}_{6}\right), 123.5\left(\mathrm{C}_{6}\right), 123.3(\mathrm{NCH}), 121.4(\mathrm{NCH}), 115.1\left(\mathrm{~d}, J_{\mathrm{CF}}=22 \mathrm{~Hz}\right.$,

$\left.\mathrm{C}_{6}\right), 88.9\left(\mathrm{C}_{5}\right), 47.6\left(\mathrm{~s},{ }^{3} J_{\mathrm{CF}}=4 \mathrm{~Hz}, \mathrm{CH}_{2}\right), 38.7\left(\mathrm{NCH}_{3}\right), 9.2\left(\mathrm{CH}_{3}\right) . \delta_{\mathrm{F}}:-112.65(1 \mathrm{~F}, \mathrm{~m})$.

\subsection{Preparation of}

$\left(\eta^{5}\right.$-pentamethylcyclopentadienyl)iridium( $\kappa C-1-(2,6$-difluorophenylmethyl)-3-methylimidazol2-ylidene) dichloride (2b)

Complex 1b $(0.099 \mathrm{~g}, 0.249 \mathrm{mmol})$ and $\left[\mathrm{Cp}^{*} \operatorname{IrCl}(\mu-\mathrm{Cl})\right]_{2}(0.100 \mathrm{~g}, 0.125 \mathrm{mmol})$ were treated as in 2.9. Yield: $0.119 \mathrm{~g},(79 \%)$. Anal. Calc. for $\mathrm{C}_{21} \mathrm{H}_{25} \mathrm{Cl}_{2} \mathrm{~F}_{2} \mathrm{IrN}_{2} .1 / 4\left(\mathrm{CHCl}_{3}\right): \mathrm{C}, 40.1 ; \mathrm{H}, 4.0$; N, 4.4. Found: C, 40.2; H, 4.4; N, 4.9\%. $[\mathrm{M}-\mathrm{Cl}]^{+}:\left[\mathrm{C}_{21} \mathrm{H}_{25}{ }^{35} \mathrm{ClF}_{2}{ }^{193} \mathrm{IrN}_{2}\right]^{+}$requires 571.1376 ; found: 571.1263. $\delta_{\mathrm{H}} 7.40\left(1 \mathrm{H}, \mathrm{m}, \mathrm{H}_{\text {para }}\right), 6.98\left(2 \mathrm{H}, \mathrm{m}, \mathrm{H}_{\text {meta }}\right), 6.88\left(1 \mathrm{H}, \mathrm{d},{ }^{3} J_{\mathrm{HH}}=\right.$ $2.2 \mathrm{~Hz}, \mathrm{HCCH}), 6.49\left(1 \mathrm{H}, \mathrm{d},{ }^{3} J_{\mathrm{HH}}=2.2 \mathrm{~Hz}, \mathrm{HCCH}\right), 5.98\left(1 \mathrm{H}, \mathrm{d},{ }^{2} J_{\mathrm{HH}}=15.3 \mathrm{~Hz}, \mathrm{NCHH}\right)$, $5.40\left(1 \mathrm{H}, \mathrm{d},{ }^{2} J_{\mathrm{HH}}=15.3 \mathrm{~Hz}, \mathrm{NCH} H\right), 4.01\left(3 \mathrm{H}, \mathrm{s}, \mathrm{NCH}_{3}\right), 1.71\left(15 \mathrm{H}, \mathrm{s}, \mathrm{CH}_{3}\right) . \delta_{\mathrm{C}} 161.9(\mathrm{dd}$, $\left.{ }^{1} J_{\mathrm{CF}}=251 \mathrm{~Hz},{ }^{3} J_{\mathrm{CF}}=7 \mathrm{~Hz}, \mathrm{CF}\right), 156.8\left(\mathrm{IrCN}_{2}\right), 132.5\left(\mathrm{t}, J_{\mathrm{CF}}=11 \mathrm{~Hz}, \mathrm{C}_{6}\right), 122.7(\mathrm{NCH})$, $120.2(\mathrm{NCH}), 111.9\left(\mathrm{dm}, J_{\mathrm{CF}}=25 \mathrm{~Hz}, \mathrm{C}_{6}\right), 89.0\left(\mathrm{C}_{5}\right), 43.5\left(\mathrm{t},{ }^{3} J_{\mathrm{CF}}=4, \mathrm{NCH}_{2}\right), 38.8\left(\mathrm{NCH}_{3}\right)$, 9.1 $\left(\mathrm{CH}_{3}\right)$. The resonance of $\mathrm{C}_{\mathrm{ipso}}$ could not be observed; it is presumed that it is obscured by a coincidental resonance. $\delta_{\mathrm{F}}-119.57(2 \mathrm{~F}, \mathrm{dd}, J=6.9,6.9 \mathrm{~Hz})$.

\subsection{Preparation of}

( $\eta^{5}$-pentamethylcyclopentadienyl)iridium( $\kappa C$-1-(2-chloro-6-fluorophenylmethyl)-3-methylimi dazol-2-ylidene) dichloride (2c)

Complex 1c $(0.101 \mathrm{~g}, 0.244 \mathrm{mmol})$ and $\left[\mathrm{Cp}^{*} \operatorname{IrCl}(\mu-\mathrm{Cl})\right]_{2}(0.096 \mathrm{~g}, 0.121 \mathrm{mmol})$ were treated as in 2.9. Yield: $0.133 \mathrm{~g}(88 \%)$. Anal. Calc. for $\mathrm{C}_{21} \mathrm{H}_{25} \mathrm{Cl}_{3} \mathrm{FIrN}_{2}: \mathrm{C}, 40.5 ; \mathrm{H}, 4.0 ; \mathrm{N}, 4.5$. Found: C, 40.0; H, 4.3; N, 4.7\%. [M - Cl $]^{+}:\left[\mathrm{C}_{21} \mathrm{H}_{25}{ }^{35} \mathrm{Cl}_{2}{ }^{193} \mathrm{FIrN}_{2}\right]^{+}$requires 587.1081; found: 587.0999. $\delta_{\mathrm{H}} 7.37\left(1 \mathrm{H}, \mathrm{dt}, J=8.0,5.9 \mathrm{~Hz}, \mathrm{C}_{6} \mathrm{H}_{3}\right), 7.33\left(1 \mathrm{H}, \mathrm{m}, \mathrm{C}_{6} \mathrm{H}_{3}\right), 7.07\left(1 \mathrm{H}, \mathrm{m}, \mathrm{C}_{6} \mathrm{H}_{3}\right)$, $6.84\left(1 \mathrm{H}, \mathrm{d},{ }^{3} J_{\mathrm{HH}}=2.0 \mathrm{~Hz}, H \mathrm{CCH}\right), 6.39\left(1 \mathrm{H}, \mathrm{d},{ }^{3} J_{\mathrm{HH}}=2.0 \mathrm{~Hz}, \mathrm{HCCH}\right), 6.30\left(1 \mathrm{H}, \mathrm{d},{ }^{2} J_{\mathrm{HH}}=\right.$ 
15.1 Hz, $\mathrm{NCHH}), 5.34\left(1 \mathrm{H}, \mathrm{d},{ }^{2} J_{\mathrm{HH}}=15.1 \mathrm{~Hz}, \mathrm{NCH} H\right), 4.01\left(3 \mathrm{H}, \mathrm{s}, \mathrm{NCH}_{3}\right), 1.71(15 \mathrm{H}, \mathrm{s}$, $\left.\mathrm{CH}_{3}\right) . \delta_{\mathrm{C}} 162.2\left(\mathrm{~d},{ }^{1} J_{\mathrm{CF}}=252 \mathrm{~Hz}, \mathrm{CF}\right), 156.3\left(\operatorname{IrCN}_{2}\right), 137.1\left(\mathrm{~d}, J_{\mathrm{CF}}=6 \mathrm{~Hz}, \mathrm{C}_{6}\right), 131.0\left(\mathrm{~d}, J_{\mathrm{CF}}\right.$ $\left.=10 \mathrm{~Hz}, \mathrm{C}_{6}\right), 126.0\left(\mathrm{~d}, J_{\mathrm{CF}}=3 \mathrm{~Hz}, \mathrm{C}_{6}\right), 122.5(\mathrm{NCH}), 121.7\left(\mathrm{~d}, J_{\mathrm{CF}}=18 \mathrm{~Hz}, \mathrm{C}_{6}\right) 120.1$ $(\mathrm{NCH}), 114.6\left(\mathrm{~d}, J_{\mathrm{CF}}=23 \mathrm{~Hz}, \mathrm{C}_{6}\right), 88.9\left(\mathrm{C}_{5}\right), 44.2\left(\mathrm{~d},{ }^{3} J_{\mathrm{CF}}=3 \mathrm{~Hz}, \mathrm{NCH}_{2}\right), 38.9\left(\mathrm{NCH}_{3}\right), 9.2$ $\left(\mathrm{CH}_{3}\right) . \delta_{\mathrm{F}}-110.01(1 \mathrm{~F}, \mathrm{~m})$.

\subsection{Preparation of}

( $\eta^{5}$-pentamethylcyclopentadienyl)iridium( $\kappa C$-1-(2-bromo-6-fluorophenylmethyl)-3-methylimi dazol-2-ylidene) dichloride (2d)

Complex 1d $(0.069 \mathrm{~g}, 0.150 \mathrm{mmol})$ and $\left[\mathrm{Cp}^{*} \operatorname{IrCl}(\mu-\mathrm{Cl})\right]_{2}(0.060 \mathrm{~g}, 0.075 \mathrm{mmol})$ were treated as in 2.9. Yield: $0.080 \mathrm{~g}(80 \%) .[\mathrm{M}-\mathrm{Cl}]^{+}:\left[\mathrm{C}_{21} \mathrm{H}_{25}{ }^{79} \mathrm{Br}^{35} \mathrm{Cl}^{193} \mathrm{FIrN}_{2}\right]^{+}$requires 631.0576; found: $631.0476 . \delta_{\mathrm{H}} 7.51\left(1 \mathrm{H}, \mathrm{dt}, J=8.2,1.0 \mathrm{~Hz}, \mathrm{C}_{6} \mathrm{H}_{3}\right), 7.30\left(1 \mathrm{H}, \mathrm{dt}, J=8.3,6.1 \mathrm{~Hz}, \mathrm{C}_{6} \mathrm{H}_{3}\right)$, $7.10\left(1 \mathrm{H}, \mathrm{ddd}, J=9.5,8.4,1.1 \mathrm{~Hz}, \mathrm{C}_{6} \mathrm{H}_{3}\right), 6.84\left(1 \mathrm{H}, \mathrm{d},{ }^{3} J_{\mathrm{HH}}=2.1 \mathrm{~Hz}, H C C H\right), 6.39(1 \mathrm{H}, \mathrm{d}$, $\left.{ }^{3} J_{\mathrm{HH}}=2.0 \mathrm{~Hz}, \mathrm{HCCH}\right), 6.31\left(1 \mathrm{H}, \mathrm{d},{ }^{2} J_{\mathrm{HH}}=14.5 \mathrm{~Hz}, \mathrm{NCHH}\right), 5.37\left(1 \mathrm{H}, \mathrm{d},{ }^{2} J_{\mathrm{HH}}=14.5 \mathrm{~Hz}\right.$, $\mathrm{NCH} H), 4.01\left(3 \mathrm{H}, \mathrm{s}, \mathrm{NCH}_{3}\right), 1.71\left(15 \mathrm{H}, \mathrm{s}, \mathrm{CH}_{3}\right) . \delta_{\mathrm{C}} 162.1\left(\mathrm{~d},{ }^{1} J_{\mathrm{CF}}=253 \mathrm{~Hz}, \mathrm{CF}\right), 156.3$ $\left(\mathrm{IrCN}_{2}\right), 131.4\left(\mathrm{~d}, J_{\mathrm{CF}}=10 \mathrm{~Hz}, \mathrm{C}_{6}\right), 129.3\left(\mathrm{~d}, J_{\mathrm{CF}}=3 \mathrm{~Hz}, \mathrm{C}_{6}\right), 127.2\left(\mathrm{~d}, J_{\mathrm{CF}}=4 \mathrm{~Hz}, \mathrm{C}_{6}\right), 123.5$ $\left(\mathrm{d}, J_{\mathrm{CF}}=18 \mathrm{~Hz}, \mathrm{C}_{6}\right), 122.4(\mathrm{NCH}), 120.2(\mathrm{NCH}), 115.2\left(\mathrm{~d}, J_{\mathrm{CF}}=23 \mathrm{~Hz}, \mathrm{C}_{6}\right), 88.9\left(\mathrm{C}_{5}\right), 49.7$ $\left(\mathrm{NCH}_{2}\right), 38.9\left(\mathrm{NCH}_{3}\right), 9.2\left(\mathrm{CH}_{3}\right) . \delta_{\mathrm{F}}-108.71(1 \mathrm{~F}, \mathrm{~m})$.

\subsection{Preparation of}

( $\eta^{5}$-pentamethylcyclopentadienyl)iridium( $\kappa C-1-(2-$ iodo-6-fluorophenylmethyl)-3-methylimida zol-2-ylidene) dichloride (2e)

Complex 1e $(0.056 \mathrm{~g}, 0.110 \mathrm{mmol})$ and $\left[\mathrm{Cp}^{*} \operatorname{IrCl}(\mu-\mathrm{Cl})\right]_{2}(0.044 \mathrm{~g}, 0.055 \mathrm{mmol})$ were treated as in 2.9. Yield: $0.067 \mathrm{~g}(85 \%) .[\mathrm{M}-\mathrm{Cl}]^{+}:\left[\mathrm{C}_{21} \mathrm{H}_{25}{ }^{35} \mathrm{ClFI}^{193} \mathrm{IrN}_{2}\right]^{+}$requires 679.0437; found: 
679.0330. $\delta_{\mathrm{H}} 7.86\left(1 \mathrm{H}, \mathrm{m}, \mathrm{C}_{6} \mathrm{H}_{3}\right), 7.12\left(2 \mathrm{H}, \mathrm{m}, \mathrm{C}_{6} \mathrm{H}_{3}\right), 6.85\left(1 \mathrm{H}, \mathrm{d} . J_{\mathrm{HH}}=2.1 \mathrm{~Hz}, H C C H\right)$, $6.38\left(1 \mathrm{H}, \mathrm{d} . J_{\mathrm{HH}}=2.1 \mathrm{~Hz}, \mathrm{HCCH}\right), 6.18(1 \mathrm{H}$, br s, $\mathrm{NCHH}), 5.47(1 \mathrm{H}$, br s, NCHH$), 4.01(3 \mathrm{H}$, $\left.\mathrm{s}, \mathrm{NCH}_{3}\right), 1.72\left(15 \mathrm{H}, \mathrm{s}, \mathrm{CH}_{3}\right) . \delta_{\mathrm{C}} 161.1\left(\mathrm{~d},{ }^{1} J_{\mathrm{CF}}=253 \mathrm{~Hz}, \mathrm{CF}\right), 156.3\left(\mathrm{IrCN}_{2}\right), 136.1\left(\mathrm{C}_{6}\right)$, $132.0\left(\mathrm{~d}, J_{\mathrm{CF}}=9 \mathrm{~Hz}, \mathrm{C}_{6}\right), 126.7\left(\mathrm{~d}, J_{\mathrm{CF}}=17 \mathrm{~Hz}, \mathrm{C}_{6}\right), 122.5(\mathrm{NCH}), 120.2(\mathrm{NCH}), 116.2(\mathrm{~d}$, $\left.J_{\mathrm{CF}}=23 \mathrm{~Hz}, \mathrm{C}_{6}\right), 103.2\left(\mathrm{~d}, J_{\mathrm{CF}}=3 \mathrm{~Hz}, \mathrm{C}_{6}\right), 88.9\left(\mathrm{C}_{5}\right), 54.7\left(\mathrm{NCH}_{2}\right), 38.9\left(\mathrm{NCH}_{3}\right), 9.2\left(\mathrm{CH}_{3}\right)$. $\delta_{\mathrm{F}}-108.27(1 \mathrm{~F}$, br s).

\subsection{Treatment of}

$\left(\eta^{5}\right.$-pentamethylcyclopentadienyl)iridium( $\kappa C-1-(2$-fluorophenylmethyl)-3-methylimidazol-2-y lidene) dichloride and ( $\eta^{5}$-pentamethylcyclopentadienyl)iridium( $\kappa C-1-(2-h a l o-6-f l u o r o p h e n y l m e t h y l)-3-m e t h y l i m i d a$ zol-2-ylidene) dichloride with silver particles.

A deuterochloroform solution $\left(c a .0 .8 \mathrm{~cm}^{3}\right)$ of $\mathbf{2 a}-\mathbf{2 e}(c a .15 \mathrm{mg})$ in an NMR tube was treated with silver nanoparticles $(\mathrm{ca} .25 \mathrm{mg})$ formed from silver nitrate and triethylamine in methanol or ethanol [12]. The tube was shaken and left overnight, after which time the NMR spectra showed the reaction had reached completion. The solution was diluted with dichloromethane $\left(5 \mathrm{~cm}^{3}\right)$ and ca. $0.2 \mathrm{~g}$ of anhydrous magnesium sulphate was added. The solution was then filtered through a glass pipette containing a plug of cotton wool and celite $(5 \mathrm{~cm})$. The celite was washed with dichloromethane $\left(5 \mathrm{~cm}^{3}\right)$. Evaporation of the solvent from the combined filtrate and washing yielded the product as a yellow oil. Recrystallization by slow diffusion of pentane into a dichloromethane solution gave yellow needles.

3b was obtained as a partial pentane solvate. Anal. Calc. for $\mathrm{C}_{21} \mathrm{H}_{25} \mathrm{ClFIrN}_{2} \cdot{ }^{1} / 4 \mathrm{C}_{5} \mathrm{H}_{12}: \mathrm{C}, 46.9 ; \mathrm{H}$, 4.95; N, 4.9. Found: C, 46.6; $\mathrm{H}, 4.7 \mathrm{~N}, 4.9 \%$. $[\mathrm{M}-\mathrm{Cl}]^{+}:\left[\mathrm{C}_{21} \mathrm{H}_{25} \mathrm{~F}^{193} \mathrm{IrN}_{2}\right]^{+}$requires 517.1704; found: $517.1650 . \delta_{\mathrm{H}} 7.40\left(1 \mathrm{H}, \mathrm{dd}, J_{\mathrm{HH}}=7.6,1.1 \mathrm{~Hz}, \mathrm{C}_{6} \mathrm{H}_{3}\right), 7.01\left(1 \mathrm{H}, \mathrm{d},{ }^{3} J_{\mathrm{HH}}=2.0 \mathrm{~Hz}\right.$, $H C C H), 6.96\left(1 \mathrm{H}, \mathrm{m}, \mathrm{C}_{6} \mathrm{H}_{3}\right), 6.93\left(1 \mathrm{H}, \mathrm{t},{ }^{3} J_{\mathrm{HH}}=2.0 \mathrm{~Hz}, \mathrm{HCCH}\right), 6.55\left(1 \mathrm{H}, \mathrm{ddd}, J_{\mathrm{FF}}=10.4\right.$ 
$\left.\mathrm{Hz}, J_{\mathrm{HH}}=8.2,1.1 \mathrm{~Hz}, \mathrm{C}_{6} \mathrm{H}_{3}\right), 5.28\left(1 \mathrm{H}, \mathrm{d},{ }^{2} J_{\mathrm{HH}}=14.4 \mathrm{~Hz}, \mathrm{CHH}\right), 4.55\left(1 \mathrm{H}, \mathrm{d},{ }^{2} J_{\mathrm{HH}}=14.4 \mathrm{~Hz}\right.$ $\mathrm{CH} H), 3.94\left(3 \mathrm{H}, \mathrm{s}, \mathrm{NCH}_{3}\right), 1.69\left(15 \mathrm{H}, \mathrm{s}, \mathrm{CH}_{3}\right) . \delta_{\mathrm{C}} 157.4\left(\mathrm{~d},{ }^{1} J_{\mathrm{CF}}=246 \mathrm{~Hz}, \mathrm{CF}\right), 157.1$ $\left(\operatorname{IrCN}_{2}\right), 148.1\left(\operatorname{IrC}\left(\mathrm{C}_{6}\right)\right), 136.8\left(\mathrm{~d}, J_{\mathrm{CF}}=3 \mathrm{~Hz}, \mathrm{C}_{6}\right), 124.8\left(\mathrm{~d}, J_{\mathrm{CF}}=9 \mathrm{~Hz}, \mathrm{C}_{6}\right), 121.4(\mathrm{NCH})$, 120.7 (NCH), $108.2\left(\mathrm{~d}, J_{\mathrm{CF}}=22 \mathrm{~Hz}, \mathrm{C}_{6}\right), 90.4\left(\mathrm{C}_{5}\right), 48.5\left(\mathrm{~d},{ }^{3} J_{\mathrm{CF}}=6 \mathrm{~Hz}, \mathrm{NCH}_{2}\right), 36.9$ $\left(\mathrm{NCH}_{3}\right), 9.4\left(\mathrm{CH}_{3}\right) . \delta_{\mathrm{F}}-123.00\left(1 \mathrm{~F}, \mathrm{dd}, J_{\mathrm{HF}}=10.4,6.1 \mathrm{~Hz}\right)$.

3c (obtained as a mixture with $3 \mathbf{b}$ from 2c) $[\mathrm{M}-\mathrm{Cl}]^{+}$: $\left[\mathrm{C}_{21} \mathrm{H}_{25}{ }^{35} \mathrm{Cl}^{193} \mathrm{IrN}_{2}\right]^{+}$requires 533.1408; found: $533.1542 . \delta_{\mathrm{H}} 7.54\left(1 \mathrm{H}, \mathrm{dd}, J_{\mathrm{HH}}=6.4,2.4 \mathrm{~Hz}, \mathrm{C}_{6} \mathrm{H}_{3}\right), 7.01\left(1 \mathrm{H}, \mathrm{d},{ }^{3} J_{\mathrm{HH}}=2.0 \mathrm{~Hz}\right.$, $H C C H), 6.96\left(2 \mathrm{H}, \mathrm{m}, \mathrm{C}_{6} \mathrm{H}_{3}\right), 6.92\left(1 \mathrm{H}, \mathrm{d},{ }^{3} J_{\mathrm{HH}}=2.0 \mathrm{~Hz}, \mathrm{HCCH}\right), 5.49\left(1 \mathrm{H}, \mathrm{d},{ }^{2} J_{\mathrm{HH}}=14.5\right.$ $\mathrm{Hz}, \mathrm{NCHH}), 4.59\left(1 \mathrm{H}, \mathrm{d},{ }^{2} J_{\mathrm{HH}}=14.5 \mathrm{~Hz}, \mathrm{NCH} H\right), 3.92\left(3 \mathrm{H}, \mathrm{s}, \mathrm{NCH}_{3}\right), 1.68\left(15 \mathrm{H}, \mathrm{s}, \mathrm{CH}_{3}\right)$. $\delta_{\mathrm{C}} 157.4\left(\mathrm{IrCN}_{2}\right), 147.8\left(\operatorname{IrC}\left(\mathrm{C}_{6}\right)\right), 140.3\left(\mathrm{C}_{6}\right), 135.3\left(\mathrm{C}_{6}\right), 128.9\left(\mathrm{C}_{6}\right), 128.5\left(\mathrm{C}_{6}\right), 122.9\left(\mathrm{C}_{6}\right)$, $121.4(\mathrm{NCH}), 120.8(\mathrm{NCH}), 90.4\left(\mathrm{C}_{5}\right), 52.7\left(\mathrm{NCH}_{2}\right), 36.9\left(\mathrm{NCH}_{3}\right), 9.4\left(\mathrm{CH}_{3}\right)$.

3d (obtained as a mixture with $3 \mathbf{b}$ from 2d) $[\mathrm{M}-\mathrm{Cl}]^{+}:\left[\mathrm{C}_{21} \mathrm{H}_{25}{ }^{79} \mathrm{Br}^{193} \operatorname{IrN}_{2}\right]^{+}$requires 577.0903; found: 577.1098. $\delta_{\mathrm{H}} 7.58\left(1 \mathrm{H}, \mathrm{dd}, J_{\mathrm{HH}}=7.5,1.1 \mathrm{~Hz}, \mathrm{C}_{6} \mathrm{H}_{3}\right), 7.06\left(1 \mathrm{H}, \mathrm{dd}, J_{\mathrm{HH}}=\right.$ 7.8, 1.2 Hz, $\left.\mathrm{C}_{6} \mathrm{H}_{3}\right), 7.01-6.92(2 \mathrm{H}, \mathrm{HCCH}), 6.82\left(1 \mathrm{H}, \mathrm{t}, J_{\mathrm{HH}}=7.6 \mathrm{~Hz}, \mathrm{C}_{6} \mathrm{H}_{3}\right), 5.48(1 \mathrm{H}, \mathrm{d}$, $\left.{ }^{2} J_{\mathrm{HH}}=14.6 \mathrm{~Hz}, \mathrm{NCHH}\right) 4.62\left(1 \mathrm{H}, \mathrm{d},{ }^{2} J_{\mathrm{HH}}=14.5 \mathrm{~Hz}, \mathrm{NCH} H\right), 3.92\left(3 \mathrm{H}, \mathrm{s}, \mathrm{NCH}_{3}\right), 1.68(15 \mathrm{H}$, $\left.\mathrm{s}, \mathrm{CH}_{3}\right) . \delta_{\mathrm{C}} 157.7\left(\mathrm{IrCN}_{2}\right), 148.2\left(\operatorname{IrC}\left(\mathrm{C}_{6}\right)\right), 141.0\left(\mathrm{C}_{6}\right), 129.0\left(\mathrm{C}_{6}\right), 126.2\left(\mathrm{C}_{6}\right), 121.4(\mathrm{NCH})$, $120.8(\mathrm{NCH}), 119.9\left(\mathrm{C}_{6}\right), 90.4\left(\mathrm{C}_{5}\right), 55.7\left(\mathrm{NCH}_{2}\right), 36.9\left(\mathrm{NCH}_{3}\right), 9.4\left(\mathrm{CH}_{3}\right)$. The resonance of one phenyl carbon atom could not be observed; it is presumed that it is obscured by a coincidental resonance.

3e (obtained as a mixture with $3 \mathbf{b}$ from $2 \mathbf{e}$ ) $[\mathrm{M}-\mathrm{Cl}]^{+}:\left[\mathrm{C}_{21} \mathrm{H}_{25} \mathrm{I}^{193} \mathrm{IrN}_{2}\right]^{+}$requires 625.0765 ; found: $625.0673 . \delta_{\mathrm{H}} 7.61\left(1 \mathrm{H}, \mathrm{dd}, J_{\mathrm{HH}}=7.5,1.2 \mathrm{~Hz}, \mathrm{C}_{6} \mathrm{H}_{3}\right), 7.35\left(1 \mathrm{H}, \mathrm{dd}, J_{\mathrm{HH}}=7.8,1.2 \mathrm{~Hz}\right.$, $\left.\mathrm{C}_{6} \mathrm{H}_{3}\right), 7.02\left(1 \mathrm{H}, \mathrm{d},{ }^{3} J_{\mathrm{HH}}=2.0 \mathrm{~Hz}, H C C H\right), 6.93\left(1 \mathrm{H}, \mathrm{d},{ }^{3} J_{\mathrm{HH}}=2.0 \mathrm{~Hz}, \mathrm{HCCH}\right), 6.65(1 \mathrm{H}, \mathrm{t}$, $\left.J_{\mathrm{HH}}=7.8 \mathrm{~Hz}, \mathrm{C}_{6} \mathrm{H}_{3}\right), 5.36\left(1 \mathrm{H}, \mathrm{d},{ }^{2} J_{\mathrm{HH}}=14.6 \mathrm{~Hz}, \mathrm{NCHH}\right), 4.69\left(1 \mathrm{H}, \mathrm{d},{ }^{2} J_{\mathrm{HH}}=14.6 \mathrm{~Hz}\right.$, $\mathrm{NCH} H), 3.93\left(3 \mathrm{H}, \mathrm{s}, \mathrm{NCH}_{3}\right), 1.68\left(15 \mathrm{H}, \mathrm{s}, \mathrm{CH}_{3}\right) . \delta_{\mathrm{C}} 158.4\left(\mathrm{IrCN}_{2}\right), 147.8\left(\operatorname{IrC}\left(\mathrm{C}_{6}\right)\right), 142.0$ 
$\left(\mathrm{C}_{6}\right), 139.9\left(\mathrm{C}_{6}\right), 133.2\left(\mathrm{C}_{6}\right), 129.6\left(\mathrm{C}_{6}\right), 121.4(\mathrm{NCH}), 120.8(\mathrm{NCH}), 96.7\left(\mathrm{C}_{6}\right), 90.4\left(\mathrm{C}_{5}\right)$, $61.3\left(\mathrm{NCH}_{2}\right), 36.8\left(\mathrm{NCH}_{3}\right), 9.4\left(\mathrm{CH}_{3}\right)$.

\section{$2.15 X$-ray crystallography}

Crystals of 2a, 2c and 2d were obtained by diffusion of pentane into a chloroform solution of the relevant complex. Crystals of $\mathbf{3 b}, \mathbf{3 b} / \mathbf{3} \mathbf{c}, \mathbf{3 b} / \mathbf{3 d}$ and $3 \mathbf{b} / \mathbf{3 e}$ were obtained by diffusion of pentane into the liquid decanted from the NMR tube reactions. Diffraction data were collected on an Agilent SuperNova, single source at offset, Atlas diffractometer with graphite-monochromated $\mathrm{Cu}-\mathrm{K}_{\alpha}$ radiation. The structures were solved using Olex2 [13] with olex2.solve [14] structure solution programme using Charge Flipping and refined with the olex2.refine [14] refinement package using Gauss-Newton minimization. The non-hydrogen atoms, except for the partial fluorine atom of $\mathbf{3 c}$, were refined with anisotropic thermal parameters. Hydrogen atom positions were added in idealized positions and a riding model with fixed thermal parameters (Uij $=1.2 \mathrm{Ueq}$ for the atom to which they are bonded (1.5 for $\left.\mathrm{CH}_{3}\right)$ ) was used for subsequent refinements. The function minimized was

$\Sigma\left[\mathrm{w}\left(\left|F_{\mathrm{o}}\right|^{2}-\left|F_{\mathrm{c}}\right|^{2}\right)\right]$ with reflection weights $w^{-1}=\left[\sigma^{2}\left|F_{\mathrm{o}}\right|^{2}+(\mathrm{g} 1 P)^{2}+(\mathrm{g} 2 P)\right]$ where $P=[\max$ $\left.\left|F_{\mathrm{o}}\right|^{2}+2\left|F_{\mathrm{c}}\right|^{2}\right] / 3$. In addition to the 8 molecules, the unit cell of $\mathbf{3 b}$ contained ca. 153 electrons, consistent with ca. 3.2 molecules of pentane, occupying a void of $682 \AA^{3}$. Attempts to model these data were unsuccessful. Consequently the data were treated with the Solvent Mask routine [14].

CCDC 1839757 (2a) 1839758 (2c), 1839759 (2d), 1839760 (3b), 1839761 (3b/3c), 1839762 (3b/3d) and 1839763 (3b/3e) contain the supplementary crystallographic data for this paper. These data can be obtained free of charge from The Cambridge Crystallographic Data Centre via www.ccdc.cam.ac.uk/data_request/cif. 


\section{Results and discussion}

The iridium complexes [Cp* $\left.\mathrm{IrCl}_{2}\left(\kappa \mathrm{C}-\mathrm{MeNC}_{3} \mathrm{H}_{2} \mathrm{NCH}_{2} \mathrm{C}_{6} \mathrm{H}_{3} \mathrm{X}-2-\mathrm{F}-6\right)\right](\mathbf{2 a} \mathrm{X}=\mathrm{H}, \mathbf{2 b}$ $\mathrm{X}=\mathrm{F}, \mathbf{2} \mathbf{c} \mathrm{X}=\mathrm{Cl}, \mathbf{2 d} \mathrm{X}=\mathrm{Br}, \mathbf{2 e} \mathrm{X}=\mathrm{I}$ ) were obtained in high yields by the treatment of $[\mathrm{Cp} * \mathrm{IrCl}(\mu-\mathrm{Cl})]_{2}$ with the silver $\mathrm{NHC}$ complexes $\left[\mathrm{AgBr}\left(\kappa \mathrm{C}-\mathrm{MeNC}_{3} \mathrm{H}_{2} \mathrm{NCH}_{2} \mathrm{C}_{6} \mathrm{H}_{3} \mathrm{X}-2-\mathrm{F}-6\right)\right]$ (1a $X=H, 1 b X=F, 1 c X=C l, 1 d X=B r, 1 e X=I)$, which were formed by treating the imidazolium salts $\left[\mathrm{MeNC}_{3} \mathrm{H}_{3} \mathrm{NCH}_{2} \mathrm{C}_{6} \mathrm{H}_{3} \mathrm{X}-2-\mathrm{F}-6\right] \mathrm{Br}$ [10] with silver(I) oxide. The complexes were characterized by NMR spectroscopy and high resolution mass spectrometry. Complexes 2a, $\mathbf{2} \mathbf{b}$ and $\mathbf{2 c}$ were also characterized by elemental analysis. The structures of complexes $\mathbf{2 a}$, 2c and 2d (Figures 1 - 3) were determined by single crystal X-ray diffraction studies.

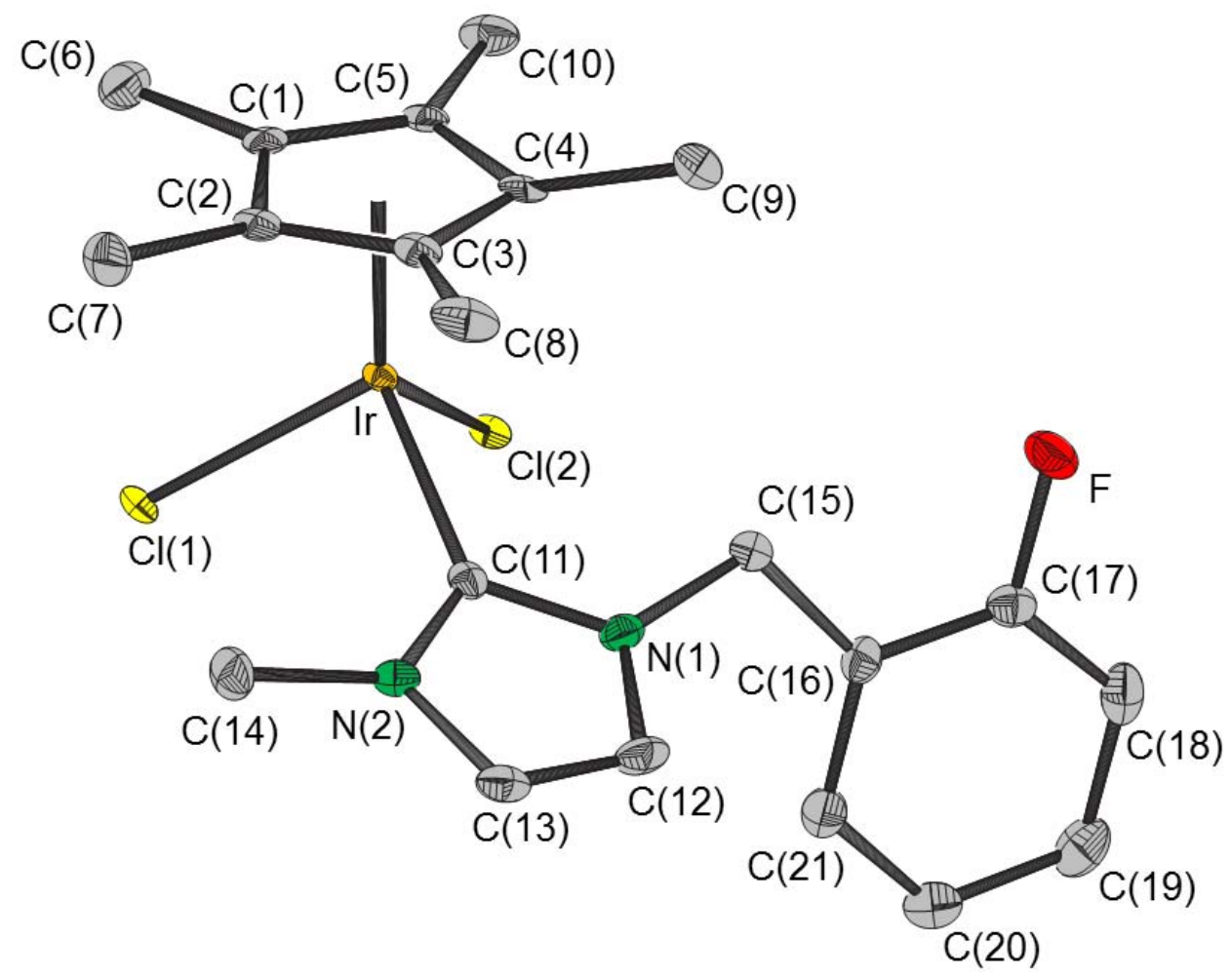

Fig. 1. Structure of

$\left(\eta^{5}\right.$-pentamethylcyclopentadienyl)iridium( $\kappa C$-1-(2-fluorophenylmethyl)-3-methylimidazol-2ylidene) dichloride (2a). Thermal ellipsoids are at the 50\% level. Hydrogen atoms are omitted for clarity. 


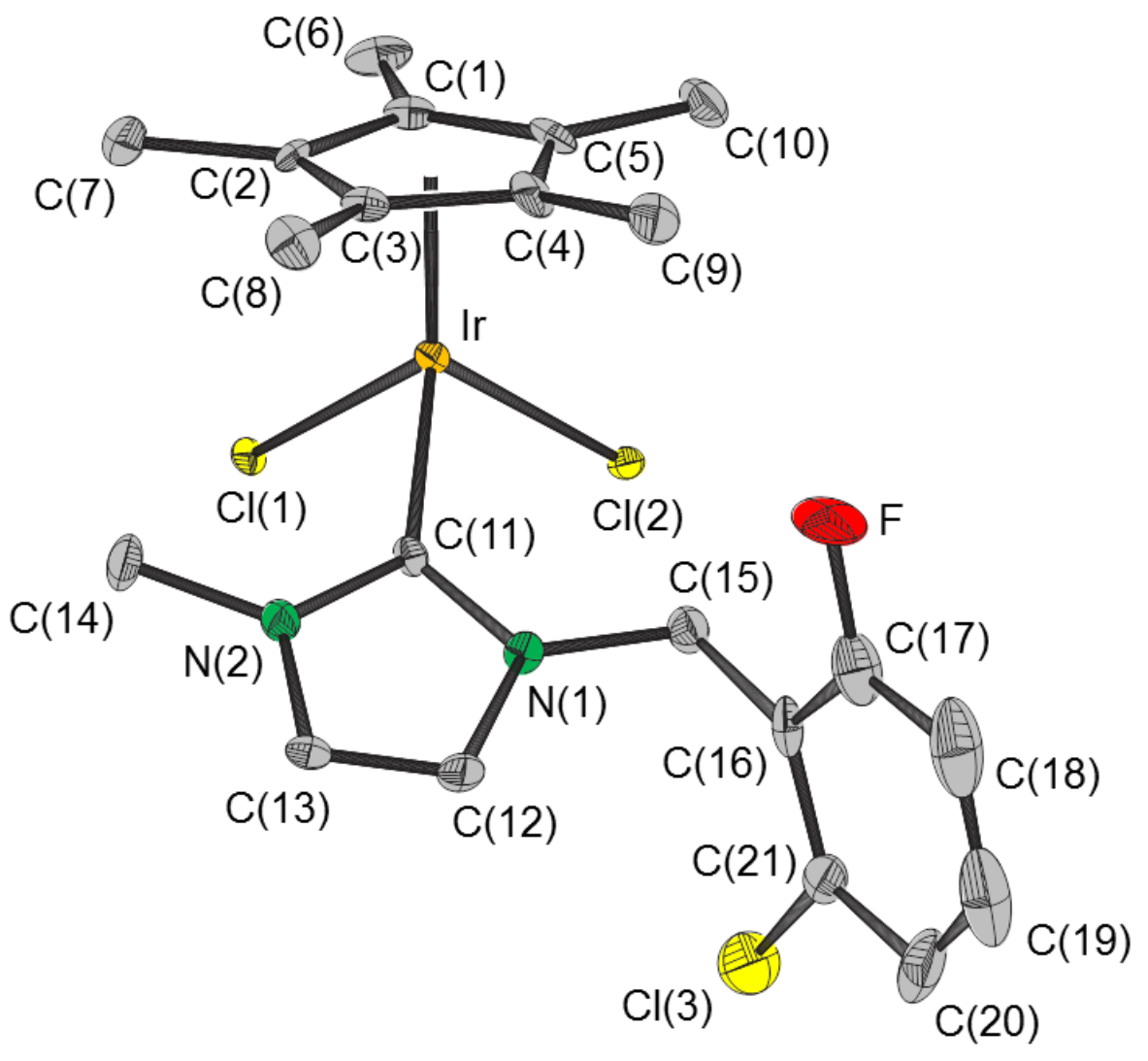

Fig. 2. Structure of

$\left(\eta^{5}\right.$-pentamethylcyclopentadienyl)iridium( $\kappa \mathrm{C}-1-(2-$ chloro-6-fluorophenylmethyl)-3-methylim idazol-2-ylidene) dichloride (2c). Thermal ellipsoids are at the 50\% level. Hydrogen atoms are omitted for clarity. 


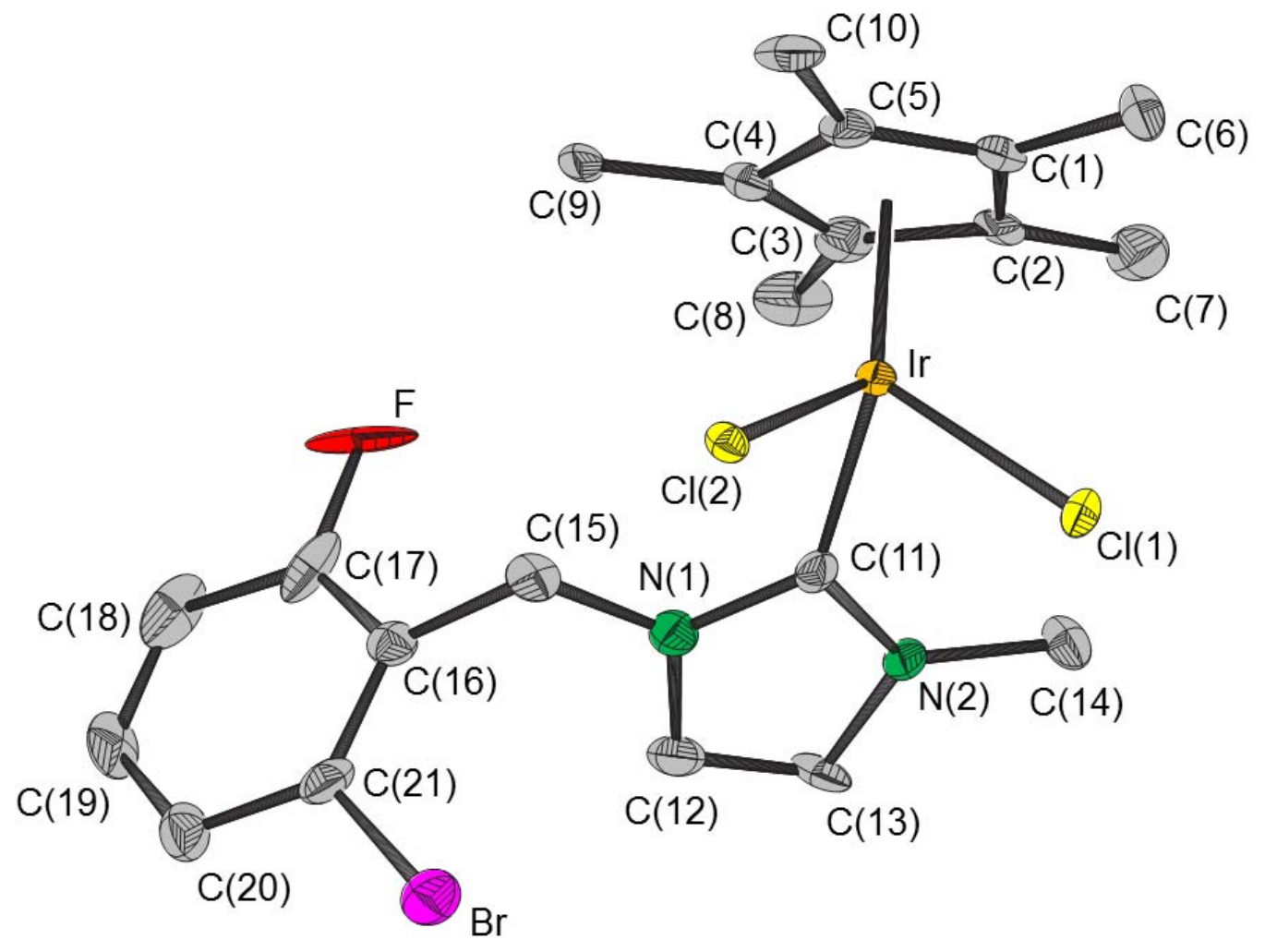

Fig. 3. Structure of

$\left(\eta^{5}\right.$-pentamethylcyclopentadienyl)iridium( $\kappa \mathrm{C}$-1-(2-bromo-6-fluorophenylmethyl)-3-methylim idazol-2-ylidene) dichloride (2d). Thermal ellipsoids are at the 50\% level. Hydrogen atoms are omitted for clarity.

In order to investigate the reactions between complexes $\mathbf{2 a}-\mathbf{2 e}$ and silver nanoparticles [12] by in situ NMR spectroscopy, experiments were performed in deuterochloroform in NMR tubes, and the final products were also analysed by mass spectrometry and single-crystal X-ray diffraction. Treatment of a solution of 2a with silver particles over 12 hours gave the $\mathrm{C}-\mathrm{H}$ bond activated product, $3 \mathbf{b}$, exclusively. Treatment of $\mathbf{2 b}$ with silver particles also gave $\mathbf{3 b}$, but by $\mathbf{C}-\mathrm{F}$ bond activation. The structure of $\mathbf{3} \mathbf{b}$ was determined by single crystal X-ray diffraction (Figure 4). The crystal structure contained additional electron density, which can be ascribed to the presence of $c a .0 .4$ molecules of 
pentane per molecule of $\mathbf{3 b}$. This could not be modelled, presumably because its position and conformation in channels parallel to the $c$ axis, which account for $c a .15 \%$ of the volume (Figure 5), are severely disordered. The presence of pentane is consistent with the elemental analysis and is also observed in the ${ }^{1} \mathrm{H}$ NMR spectrum.

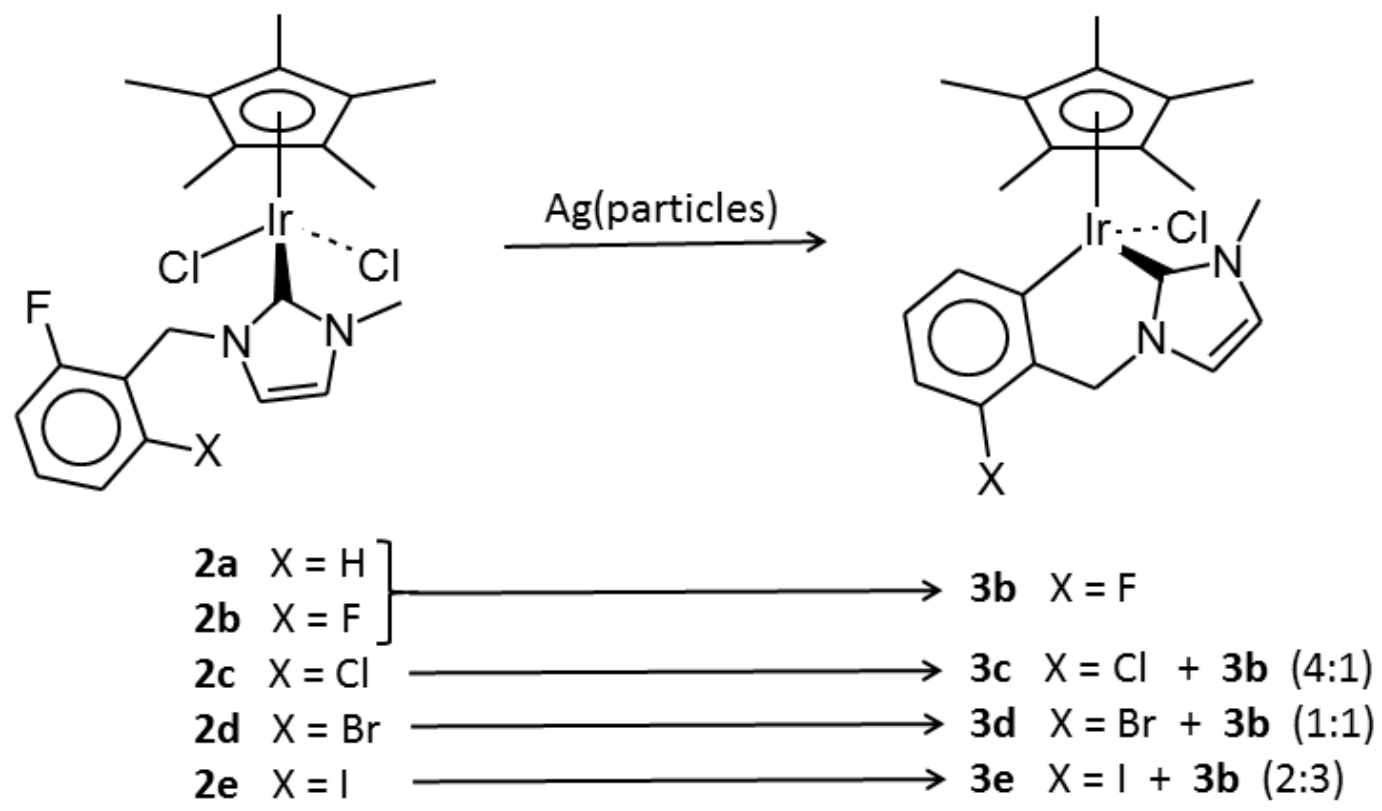

Scheme. Reaction of

( $\eta^{5}$-pentamethylcyclopentadienyl)iridium( $\kappa \mathrm{C}-1-(2-$ fluorophenylmethyl)-3-methylimidazol-2ylidene) dichloride complexes with silver particles. 


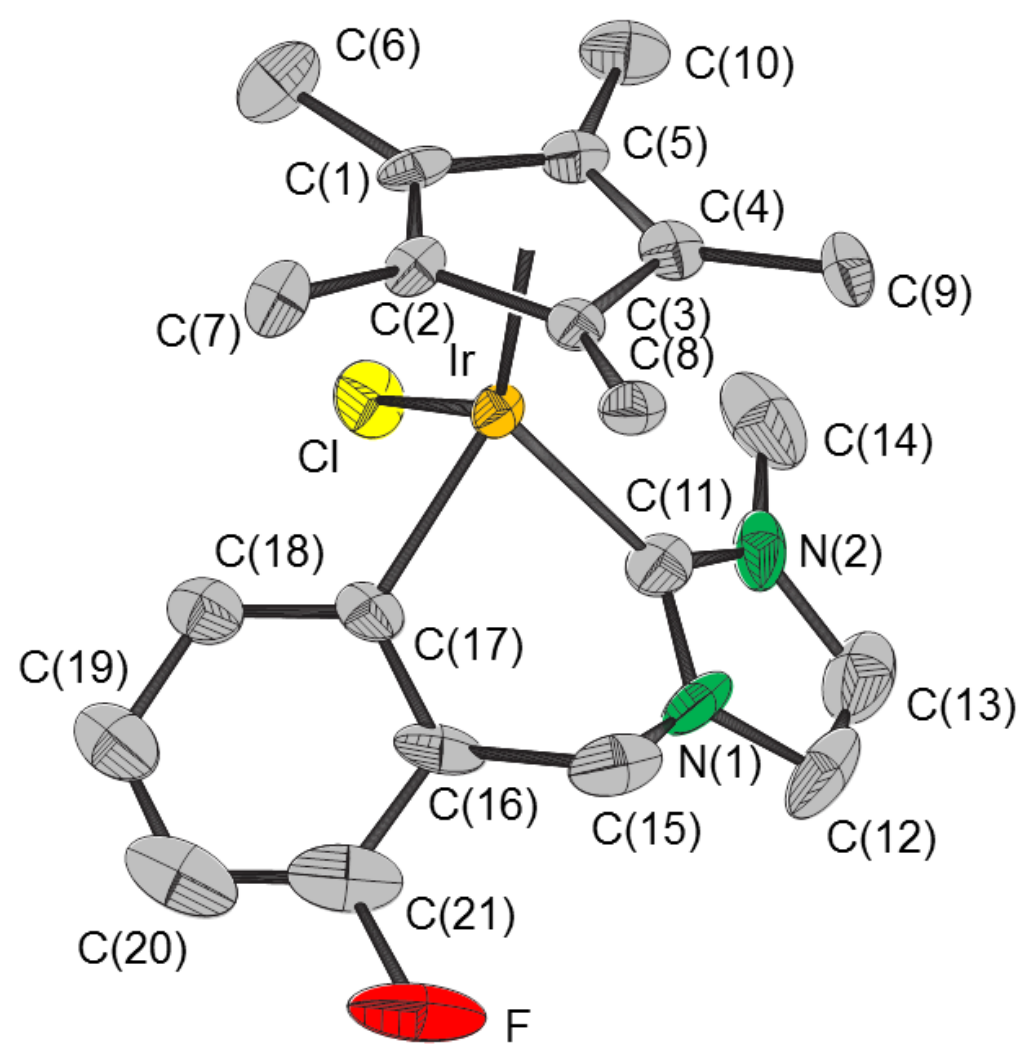

Fig. 4. Structure of

$\left(\eta^{5}\right.$-pentamethylcyclopentadienyl)iridium( $\kappa^{2} \mathrm{C}-1$-(2-fluorophenylmethyl)-3-methylimidazol-2 -ylidene) chloride (3b). Thermal ellipsoids are at the 50\% level. Hydrogen atoms are omitted for clarity. 


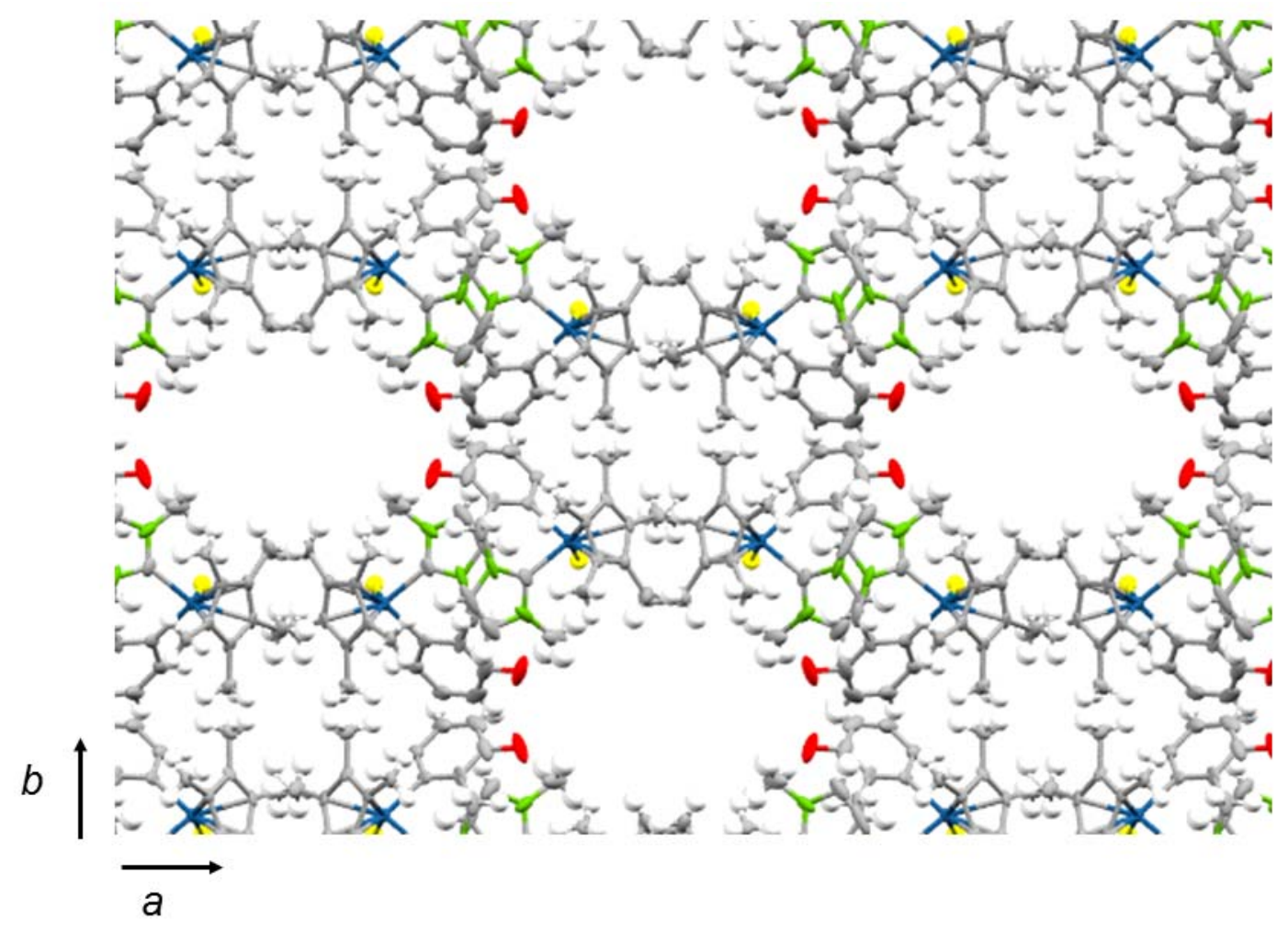

Fig. 5. Crystal structure of

( $\eta^{5}$-pentamethylcyclopentadienyl)iridium( $\kappa^{2} \mathrm{C}-1$-(2-fluorophenylmethyl)-3-methylimidazol-2 -ylidene) chloride (3b) showing the channels parallel to the $c$ axis.

On treatment with silver particles complexes $\mathbf{2 c}$, $2 \mathbf{d}$ and $\mathbf{2 e}$ underwent $\mathrm{C}-\mathrm{X}$ and $\mathrm{C}-$ F bond activation to yield mixtures of $\mathbf{3 b}$ and $\mathbf{3 c}, \mathbf{3} \mathbf{b}$ and $\mathbf{3 d}$, and $\mathbf{3 b}$ and $\mathbf{3 e}$ in $c a .1: 4,1: 1$ and 3:2 ratio respectively as evidenced by NMR spectroscopy. Mass spectrometry indicated that there was no exchange of the carbon bound halogen atom of $\mathbf{3} \mathbf{d}$ and $\mathbf{3} \mathbf{e}$ for chlorine, an atom of which is lost as chloride from the metal centre, and it is therefore surmised that exchange of atoms at this site does not occur in any of the reactions or for any of the halogens. Crystals were obtained from the reactions and the structures of the products, which co-crystallized, were elucidated by single-crystal X-ray diffraction studies. The co-crystals are isomorphous with those of $\mathbf{3 b}$ and the compounds $\mathbf{3 b}$ - $\mathbf{3 e}$ are isostructural. The structure of the co-crystal 
of $\mathbf{3 b}$ and 3d is shown in Figure 6. (The structures of $\mathbf{3 b}$ and $\mathbf{3} \mathbf{c}$, and $\mathbf{3 b}$ and $\mathbf{3 e}$ are contained in the supplementary material.) The crystal structures of the co-crystals were modelled with 3b:3c, 3b:3d and 3b:3e ratios of 44:56, $43: 57$ and 64:36 respectively, without the inclusion of solvent, although residual electron density suggested the presence of a small amount $(<0.1$ molecule per molecule of product). The bond distances and angles around iridium (Table 2) are similar to those of $\left[\mathrm{Cp} * \operatorname{IrCl}\left(\kappa \mathrm{C}^{2}-\mathrm{MeNC}_{3} \mathrm{H}_{2} \mathrm{NCH}_{2} \mathrm{C}_{6} \mathrm{~F}_{4}\right)\right][9]$.

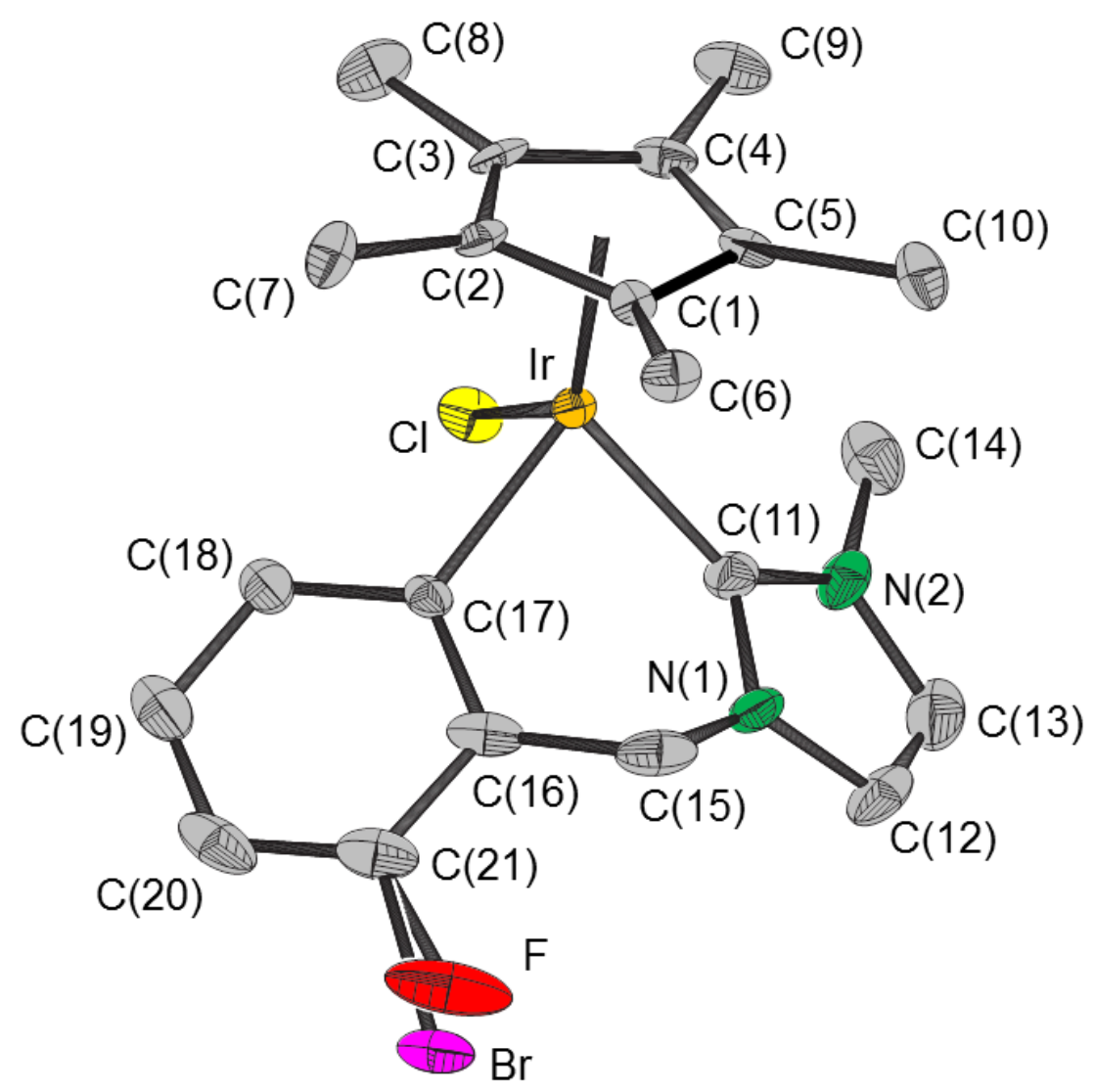

Fig. 6. Structure of the product from the co-crystal of

$\left(\eta^{5}\right.$-pentamethylcyclopentadienyl)iridium( $\kappa C^{2}-1-(2$-fluorophenylmethyl)-3-methylimidazol-2 -ylidene) chloride (3b) and

$\left(\eta^{5}\right.$-pentamethylcyclopentadienyl)iridium( $\kappa C^{2}-1$-(2-bromophenylmethyl)-3-methylimidazol-2 -ylidene) chloride (3d). Thermal ellipsoids are at the 50\% level. Hydrogen atoms are omitted for clarity. 
Since $\mathrm{C}-\mathrm{H}$ and $\mathrm{C}-\mathrm{F}$ bonds possess opposite polarity, and base induces

orthometallation involving $\mathrm{C}-\mathrm{H}$, but not $\mathrm{C}-\mathrm{F}$, bond activation [1-4,15], it is suggested that the exclusive preference for $\mathrm{C}-\mathrm{H}$ bond activation over $\mathrm{C}-\mathrm{F}$ bond activation in the reaction of $\mathbf{2 a}$ is a consequence of different mechanisms for the reaction, rather than a consequence of the relative strengths of the $\mathrm{C}-\mathrm{H}$ and $\mathrm{C}-\mathrm{F}$ bonds $\left(\mathrm{C}_{6} \mathrm{H}_{5}-\mathrm{H} 466 \mathrm{~kJ} \mathrm{~mol}^{-1}, \mathrm{C}_{6} \mathrm{H}_{5}-\mathrm{F} 526 \mathrm{~kJ}\right.$ $\left.\mathrm{mol}^{-1}[16]\right)$. The exclusive generation of $3 \mathbf{b}$ indicates that the reaction involving $\mathrm{C}-\mathrm{H}$ bond activation is significantly more rapid than that involving $\mathrm{C}-\mathrm{F}$ bond activation. The increasing ratios of $\mathrm{C}-\mathrm{X}$ to $\mathrm{C}-\mathrm{F}$ bond activation for $\mathbf{2 c}, \mathbf{2 d}$ and $2 \mathbf{e}$ correlates with the decreasing $\mathrm{C}-\mathrm{X}$ bond strength $\left(\mathrm{C}_{6} \mathrm{H}_{5}-\mathrm{Cl} 400 \mathrm{~kJ} \mathrm{~mol}^{-1}, \mathrm{C}_{6} \mathrm{H}_{5}-\mathrm{Br} 337 \mathrm{~kJ} \mathrm{~mol}^{-1}, \mathrm{C}_{6} \mathrm{H}_{5}-\mathrm{I}\right.$ $\left.274 \mathrm{~kJ} \mathrm{~mol}^{-1}[16]\right)$. However, the greater strength of the $\mathrm{C}-\mathrm{F}$ bond compared to the $\mathrm{C}-\mathrm{Cl}$ bond indicates that bond strength alone cannot account for the preference for which bond is activated.

\section{Conclusion}

Orthometallation occurs exclusively by carbon-hydrogen bond activation when $\left(\eta^{5}\right.$-pentamethylcyclopentadienyl)iridium( $\kappa C$-1-(2-fluorophenylmethyl)-3-methylimidazol-2ylidene) dichloride (2a) is treated with silver particles. The same product is formed from ( $\eta^{5}$-pentamethylcyclopentadienyl)iridium( $\kappa \mathrm{C}-1-(2,6$-fluorophenylmethyl)-3-methylimidazol2-ylidene) dichloride by carbon-fluorine bond activation. When

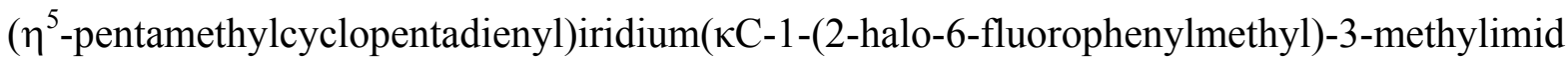
azol-2-ylidene) dichlorides (2c - 2e) are treated with silver particles, orthometallation by carbon-halogen bond activation occurs giving a mixture of two products. The identities of the four products have been confirmed by single-crystal X-ray diffraction. 


\section{References}

[1] R. Corberán, M. Sanaú, E. Peris, Highly Stable Cp*-Ir(III) Complexes with $N$ Heterocyclic Carbene Ligands as $\mathrm{C}-\mathrm{H}$ Activation Catalysts for the Deuteration of Organic Molecules, J. Am. Chem. Soc. 128 (2006) 3974-3979.

[2] R. Corberán, M. Sanaú, E. Peris, Aliphatic and Aromatic Intramolecular C-H Activation on Cp*Ir(NHC) Complexes, Organometallics 25 (2006) 4002-4008.

[3] R. Corberán, V. Lillo, J.A. Mata, E. Fernandez, E. Peris, Enantioselective Preparation of a Chiral-at-Metal Cp* $\operatorname{Ir}(\mathrm{NHC})$ Complex and Its Application in the Catalytic Diboration of Olefins, Organometallics 26 (2007) 4350-4353.

[4] A. Pontes da Costa, M. Sanaú, E. Peris, B. Royo, Easy preparation of Cp*functionalized N-heterocyclic carbenes and their coordination to rhodium and iridium, Dalton Trans. (2009) 6960-6966.

[5] Y. Tanabe, F. Hanasaka, K. Fujita, R. Yamaguchi, Scope and Mechanistic Studies of

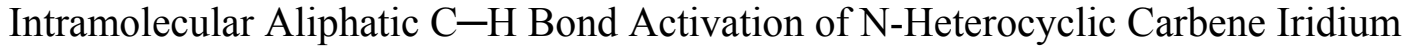
Complexes, Organometallics 26 (2007) 4618-4626.

[6] M. Benítez, E. Mas-Marzá, J.A. Mata, E. Peris, Intramolecular Oxidation of the Alcohol Functionalities in Hydroxyalkyl-N-Heterocyclic Carbene Complexes of Iridium and Rhodium, Chem. Eur. J. 17 (2011) 10453-10461.

[7] J. Holmes, C.M. Pask, C.E. Willans, Chelating N-heterocyclic carbene-carboranes offer flexible ligand coordination to $\mathrm{Ir}^{\mathrm{III}}, \mathrm{Rh}^{\mathrm{III}}$ and $\mathrm{Ru}^{\mathrm{II}}$ : effect of ligand cyclometallation in catalytic transfer hydrogenation, Dalton Trans. 45 (2016) $15818-15827$. 
[8] M. Prinz, M. Grosche, E. Herdtweck, W.A. Herrmann, Unsymmetrically Substituted Iridium(III)-Carbene Complexes by a CH-Activation Process, Organometallics 19 (2000) 1692-1694.

[9] H.P. Thomas, Y.-M. Wang, F. Lorenzini, S.J. Coles, P.N. Horton, A.C. Marr, G.C. Saunders, Cyclometallation via Carbon-Fluorine Bond Activation induced by Silver Particles, Organometallics 36 (2017) 960-963.

[10] F. Lorenzini, A.C. Marr, G.C. Saunders, H.P. Thomas, The structures of 1-(2-halo-6-fluorophenylmethyl)-1-methylimidazolium bromide salts, J. Fluor. Chem. $210(2018) 102-111$.

[11] C. White, A. Yates, P.M. Maitlis, ( $\eta^{5}$-Pentamethylcyclopentadienyl)Rhodium and Iridium Compounds, Inorg. Synth. 29 (1992) 228-234.

[12] J.-T. Wu, S. L.-C. Hsu, Preparation of triethylamine stabilized silver nanoparticles for low-temperature sintering, J. Nanopart. Res. 13 (2011) 3877-3883.

[13] O.V. Dolomanov, L.J. Bourhis, R.J. Gildea, J.A.K. Howard, H. Puschmann, OLEX2: a complete structure solution, refinement and analysis program, J. Appl. Cryst. 42 (2009) $339-341$.

[14] L.J. Bourhis, O.V. Dolomanov, R.J. Gildea, J.A.K. Howard, H. Puschmann, The anatomy of a comprehensive constrained, restrained refinement program for the modern computing environment - Olex2 dissected, Acta Crystallogr. A71 (2015) 59-75.

[15] S.J. McGrandle, G.C. Saunders, Group 9 complexes of an N-heterocycle carbene bearing a pentafluorobenzyl substituent: Attempted dehydrofluorinative coupling of cyclopentadienyl and N-heterocycle carbene ligands, J. Fluorine Chem. 126 (2005) 451-455.

[16] D.F. McMillen, D.M. Golden, Hydrocarbon bond dissociation energies, Ann, Rev. Phys. Chem. 33 (1982) 493-532. 


\section{Table 1}

Crystallographic data for ( $\eta^{5}$-pentamethylcyclopentadienyl)iridium( $\kappa \mathrm{C}-1-(2$-fluorophenylmethyl)-3-methylimidazol-2-ylidene) dichloride, 2a,

$\left(\eta^{5}\right.$-pentamethylcyclopentadienyl)iridium( $\kappa \mathrm{C}-1$-(2-chloro-6-fluorophenylmethyl)-3-methylimidazol-2-ylidene) dichloride, 2c,

( $\eta^{5}$-pentamethylcyclopentadienyl)iridium( $\kappa \mathrm{C}-1$-(2-bromo-6-fluorophenylmethyl)-3-methylimidazol-2-ylidene) dichloride, 2d,

( $\eta^{5}$-pentamethylcyclopentadienyl)iridium( $\kappa C^{2}-1$-(2-fluorophenylmethyl)-3-methylimidazol-2-ylidene) chloride, 3b, and co-crystals with

$\left(\eta^{5}\right.$-pentamethylcyclopentadienyl)iridium( $\mathrm{\kappa C}^{2}$-1-(2-chlorophenylmethyl)-3-methylimidazol-2-ylidene) chloride, 3c, with

( $\eta^{5}$-pentamethylcyclopentadienyl)iridium( $\left(\mathrm{KC}^{2}\right.$-1-(2-bromophenylmethyl)-3-methylimidazol-2-ylidene) chloride, 3d, and with

( $\eta^{5}$-pentamethylcyclopentadienyl)iridium( $\kappa C^{2}-1$-(2-iodophenylmethyl)-3-methylimidazol-2-ylidene) chloride, $3 \mathbf{e}^{2}$

\begin{tabular}{|c|c|c|c|c|c|c|c|}
\hline & $2 a$ & 2c & $2 d$ & $3 \mathbf{b}$ & $3 \mathbf{b} / 3 \mathbf{c}$ & $3 \mathbf{b} / \mathbf{3 d}$ & $3 \mathbf{b} / 3 \mathbf{e}$ \\
\hline $\begin{array}{l}\text { Formula } \\
\text { weight }\end{array}$ & 588.57 & 623.02 & 667.47 & 552.11 & 561.33 & 587.13 & 590.96 \\
\hline $\begin{array}{l}\text { Crystal } \\
\text { system }\end{array}$ & orthorhombic & orthorhombic & orthorhombic & orthorhombic & orthorhombic & orthorhombic & orthorhombic \\
\hline $\begin{array}{l}\text { Space } \\
\text { group }\end{array}$ & Pbca & $P$ na $2_{1}$ & $P$ na $2_{1}$ & Iba2 & Iba2 & Iba2 & Iba2 \\
\hline
\end{tabular}




\begin{tabular}{|c|c|c|c|c|c|c|c|}
\hline$a, \AA$ & $8.65896(14)$ & $17.8456(4)$ & $21.4894(5)$ & $26.8949(6)$ & $27.0311(5)$ & $19.5638(3)$ & $26.7156(6)$ \\
\hline$b, \AA$ & $18.7242(3)$ & $12.5932(2)$ & $9.5002(2)$ & $19.5780(4)$ & $19.4538(4)$ & $26.8744(5)$ & $19.9311(5)$ \\
\hline$c, \AA$ & $25.2239(4)$ & $9.50997(17)$ & $10.7341(2)$ & $8.48341(19)$ & $8.47896(15)$ & $8.49006(19)$ & $8.4836(2)$ \\
\hline$V, \AA^{3}$ & $4089.61(12)$ & $2137.21(7)$ & 2191.41(8) & $4466.92(17)$ & $4458.72(15)$ & $4463.77(15)$ & $4517.29(19)$ \\
\hline$Z$ & 8 & 4 & 4 & 8 & 8 & 8 & 8 \\
\hline$D_{\mathrm{c}}\left(\mathrm{g} \mathrm{cm}^{-3}\right)$ & 1.912 & 1.936 & 2.0230 & 1.642 & 1.672 & 1.747 & 1.738 \\
\hline $\begin{array}{l}\text { Crystal size } \\
\left(\mathrm{mm}^{3}\right)\end{array}$ & $\begin{array}{l}0.204 \times 0.058 \\
\times 0.037\end{array}$ & $\begin{array}{l}0.155 \times 0.043 \\
\times 0.040\end{array}$ & $\begin{array}{l}0.106 \times 0.042 \times \\
0.030\end{array}$ & $\begin{array}{l}0.790 \times 0.117 \\
\times 0.069\end{array}$ & $\begin{array}{l}0.200 \times 0.077 \times \\
0.046\end{array}$ & $0.165 \times 0.084 \times 0.026$ & $\begin{array}{l}0.219 \times 0.096 \times \\
0.057\end{array}$ \\
\hline$\mu\left(\mathrm{mm}^{-1}\right)$ & 15.186 & 15.697 & 16.232 & 12.785 & 13.386 & 13.959 & 16.482 \\
\hline$\theta$ range $\left({ }^{\circ}\right)$ & $3.48-73.90$ & $4.30-73.92$ & $4.11-74.00$ & $6.57-73.75$ & $6.55-73.80$ & $4.52-73.91$ & $4.44-73.72$ \\
\hline $\begin{array}{l}\text { Total } \\
\text { reflections }\end{array}$ & 11,857 & 10,700 & 6,491 & 11,337 & 6,809 & 11,566 & 13,285 \\
\hline $\begin{array}{l}\text { Unique } \\
\text { reflections } \\
\left(R_{\text {int }}\right)\end{array}$ & $4,001(0.0233)$ & $4,135(0.0373)$ & $3,182(0.0359)$ & $4,022(0.0324)$ & $3,621(0.0221)$ & $4,043(0.0231)$ & $4,360(0.0305)$ \\
\hline $\begin{array}{l}\text { Observed } \\
\text { reflections }\end{array}$ & 3,445 & 3,710 & 2,930 & 3,743 & 3,248 & 3,808 & 4,096 \\
\hline
\end{tabular}




\begin{tabular}{|c|c|c|c|c|c|c|c|}
\hline$[I>2 \sigma(I)]$ & & & & & & & \\
\hline $\begin{array}{l}\text { Final } R \\
\text { indices }[I> \\
2 \sigma(I)]\end{array}$ & $\begin{array}{l}R_{1}=0.0253, \\
w R_{2}=0.685\end{array}$ & $\begin{array}{l}R_{1}=0.0323, \\
w R_{2}=0.0885\end{array}$ & $\begin{array}{l}R_{1}=0.0311 \\
w R_{2}=0.0720\end{array}$ & $\begin{array}{l}R_{1}=0.0240 \\
w R_{2}=0.0604\end{array}$ & $\begin{array}{l}R_{1}=0.0335 \\
w R_{2}=0.0841\end{array}$ & $\begin{array}{l}R_{1}=0.0229 \\
w R_{2}=0.0590\end{array}$ & $\begin{array}{l}R_{1}=0.0349 \\
w R_{2}=0.0946\end{array}$ \\
\hline $\begin{array}{l}\text { Weighting } \\
\text { scheme }\end{array}$ & $\begin{array}{l}w=1 /\left[\sigma^{2}\left(F_{\mathrm{o}}\right)^{2}\right. \\
+\left\{0.0440\left(F_{\mathrm{o}}^{2}\right.\right. \\
\left.\left.+2 F_{\mathrm{c}}^{2}\right) / 3\right\}^{2}+ \\
8.0600\left(F_{\mathrm{o}}{ }^{2}+\right. \\
\left.\left.2 F_{\mathrm{c}}{ }^{2}\right) / 3\right]\end{array}$ & $\begin{array}{l}w=1 /\left[\sigma^{2}\left(F_{\mathrm{o}}\right)^{2}\right. \\
+\left\{0.0600\left(F_{\mathrm{o}}^{2}\right.\right. \\
\left.\left.+2 F_{\mathrm{c}}{ }^{2}\right) / 3\right\}^{2}+ \\
3.3550\left(F_{\mathrm{o}}^{2}+\right. \\
\left.\left.2 F_{\mathrm{c}}{ }^{2}\right) / 3\right]\end{array}$ & $\begin{array}{l}w=1 /\left[\sigma^{2}\left(F_{\mathrm{o}}\right)^{2}+\right. \\
\left\{0.0300\left(F_{\mathrm{o}}^{2}+\right.\right. \\
\left.\left.2{F_{\mathrm{c}}}^{2}\right) / 3\right\}^{2}+ \\
5.2490\left(F_{\mathrm{o}}^{2}+\right. \\
\left.\left.2{F_{\mathrm{c}}}^{2}\right) / 3\right]\end{array}$ & $\begin{array}{l}w=1 /\left[\sigma^{2}\left(F_{\mathrm{o}}\right)^{2}\right. \\
+\left\{0.0360\left(F_{\mathrm{o}}^{2}\right.\right. \\
\left.\left.\left.+2 F_{\mathrm{c}}^{2}\right) / 3\right\}^{2}\right]\end{array}$ & $\begin{array}{l}w=1 /\left[\sigma^{2}\left(F_{\mathrm{o}}\right)^{2}+\right. \\
\left\{0.0350\left(F_{\mathrm{o}}^{2}+\right.\right. \\
\left.\left.2 F_{\mathrm{c}}^{2}\right) / 3\right\}^{2}+88.7070 \\
\left.\left({F_{\mathrm{o}}}^{2}+2{F_{\mathrm{c}}}^{2}\right) / 3\right]\end{array}$ & $\begin{array}{l}w=1 /\left[\sigma^{2}\left(F_{\mathrm{o}}\right)^{2}+\right. \\
\left\{0.0400\left(F_{\mathrm{o}}^{2}+\right.\right. \\
\left.\left.2 F_{\mathrm{c}}^{2}\right) / 3\right\}^{2}+4.4700 \\
\left.\left({F_{\mathrm{o}}}^{2}+2{F_{\mathrm{c}}}^{2}\right) / 3\right]\end{array}$ & $\begin{array}{l}w=1 /\left[\sigma^{2}\left(F_{\mathrm{o}}\right)^{2}+\right. \\
\left\{0.0580\left(F_{\mathrm{o}}^{2}+\right.\right. \\
\left.\left.2 F_{\mathrm{c}}^{2}\right) / 3\right\}^{2}+37.4550 \\
\left.\left(F_{\mathrm{o}}^{2}+2 F_{\mathrm{c}}^{2}\right) / 3\right]\end{array}$ \\
\hline $\begin{array}{l}\text { Max., min. } \\
\Delta \rho\left(\mathrm{e}^{-3}\right)\end{array}$ & $1.117,-1.553$ & $0.878,-1.948$ & $1.936,-0.986$ & $0.866,-0.792$ & $1.157,-0.975$ & $0.555,-0.750$ & $2.389,-1.128$ \\
\hline $\begin{array}{l}\text { Goodness } \\
\text { of fit on } F^{2}\end{array}$ & 1.017 & 1.027 & 1.039 & 1.026 & 1.051 & 1.035 & 1.030 \\
\hline Flack & - & $0.004(17)$ & $0.014(15)$ & $-0.005(15)$ & $-0.01(2)$ & $0.015(14)$ & $0.006(16)$ \\
\hline
\end{tabular}




\begin{tabular}{|l|l|l|l|l|l|l|}
\hline parameter & & & & & & \\
\hline & & & & & & \\
\hline
\end{tabular}

${ }^{a}$ Estimated standard deviations are given in parentheses. 
Table 2 Selected distances $(\AA)$ and angles $\left({ }^{\circ}\right)$ for for $\left(\eta^{5}\right.$-pentamethylcyclopentadienyl)iridium( $(\kappa C-1-(2$-fluorophenylmethyl)-3-methylimidazol-2-ylidene) dichloride, 2a, ( $\eta^{5}$-pentamethylcyclopentadienyl)iridium( $\mathrm{\kappa C}$-1-(2-chloro-6-fluorophenylmethyl)-3-methylimidazol-2-ylidene) dichloride, 2c,

( $\eta^{5}$-pentamethylcyclopentadienyl)iridium( $\kappa \mathrm{C}-1$-(2-bromo-6-fluorophenylmethyl)-3-methylimidazol-2-ylidene) dichloride, 2d,

$\left(\eta^{5}\right.$-pentamethylcyclopentadienyl)iridium( $\kappa^{2} \mathrm{C}^{2}$-1-(2-fluorophenylmethyl)-3-methylimidazol-2-ylidene) chloride, 3b, and co-crystals with

( $\eta^{5}$-pentamethylcyclopentadienyl)iridium( $\kappa C^{2}$-1-(2-chlorophenylmethyl)-3-methylimidazol-2-ylidene) chloride, 3c, with

( $\eta^{5}$-pentamethylcyclopentadienyl)iridium( $\kappa C^{2}$-1-(2-bromophenylmethyl)-3-methylimidazol-2-ylidene) chloride, 3d, and with

( $\eta^{5}$-pentamethylcyclopentadienyl)iridium( $\mathrm{\kappa C}^{2}$-1-(2-iodophenylmethyl)-3-methylimidazol-2-ylidene) chloride, 3e. ${ }^{\mathrm{a}}$

\begin{tabular}{|c|c|c|c|c|c|c|c|}
\hline & $2 a$ & $2 c$ & $2 d$ & $\mathbf{3 b}$ & $3 \mathbf{b} / 3 \mathbf{c}$ & $3 b / 3 d$ & $3 \mathbf{b} / 3 \mathbf{e}$ \\
\hline $\mathrm{Ir}-\mathrm{Cl}(1)$ & $2.4440(9)$ & $2.445(1)$ & $2.418(2)$ & $2.4173(9)$ & $2.422(2)$ & $2.4251(8)$ & $2.4176(13)$ \\
\hline $\mathrm{Ir}-\mathrm{Cl}(2)$ & $2.4366(9)$ & $2.442(1)$ & $2.435(1)$ & - & - & - & - \\
\hline $\mathrm{Ir}-\mathrm{C}(11)$ & $2.041(4)$ & $2.054(5)$ & $2.026(9)$ & $2.029(5)$ & $2.000(9)$ & $2.006(4)$ & $1.986(7)$ \\
\hline $\mathrm{C}-\mathrm{F}$ & $1.372(5)$ & $1.376(9)$ & $1.34(1)$ & $1.380(7)$ & $1.47(2)$ & $1.44(1)$ & $1.52(2)$ \\
\hline $\mathrm{C}-\mathrm{X}(\mathrm{X})$ & - & $1.681(7)(\mathrm{Cl})$ & $1.876(9)(\mathrm{Br})$ & - & $1.78(1)(\mathrm{Cl})$ & $1.896(5)(\mathrm{Br})$ & $2.086(9)(\mathrm{I})$ \\
\hline
\end{tabular}




\begin{tabular}{|l|l|l|l|l|l|l|l|}
\hline & & & & & & \\
\hline $\mathrm{Cp} *-\mathrm{Ir}-\mathrm{Cl}(1)$ & $122.1(1)$ & $122.9(2)$ & $125.6(2)$ & $122.9(1)$ & $122.5(2)$ & $122.6(1)$ & $122.3(2)$ \\
\hline $\mathrm{Cp} \mathrm{p}^{*}-\mathrm{Ir}-\mathrm{Cl}(2)$ & $125.1(1)$ & $124.1(2)$ & $123.5(2)$ & - & - & - & - \\
\hline $\mathrm{Cp} \mathrm{p}^{*}-\mathrm{Ir}-\mathrm{C}(11)$ & $126.1(2)$ & $129.6(2)$ & $127.71(2)$ & $128.3(2)$ & $129.3(3)$ & $128.8(2)$ & $128.9(3)$ \\
\hline $\mathrm{Cp} \mathrm{p}^{*}-\mathrm{Ir}-\mathrm{C}(17)$ & - & - & - & $128.2(2)$ & $128.0(3)$ & $128.4(2)$ & $128.7(3)$ \\
\hline & & & & & & \\
\hline
\end{tabular}

${ }^{\mathrm{a}}$ Estimated standard deviations are given in parentheses. 